\title{
A Generalization of the Kostka-Foulkes Polynomials
}

\author{
ANATOL N. KIRILLOV \\ kirillov@ kurims.kyoto-u.ac.jp; Kirillov@math.nagoya-u.ac.jp \\ Research Institute for Mathematical Sciences, Kyoto University, Kyoto 606-8502, Japan \\ MARK SHIMOZONO \\ mshimo@math.vt.edu \\ Department of Mathematics, Virginia Polytechnic Institute and State University, Blacksburg, VA 24061, USA
}

Received March 24, 1998; Revised August 7, 2001; Accepted August 14, 2001

Abstract. Combinatorial objects called rigged configurations give rise to $q$-analogues of certain LittlewoodRichardson coefficients. The Kostka-Foulkes polynomials and two-column Macdonald-Kostka polynomials occur as special cases. Conjecturally these polynomials coincide with the Poincaré polynomials of isotypic components of certain graded $G L(n)$-modules supported in a nilpotent conjugacy class closure in $g l(n)$.

Keywords: generalized Kostka polynomials, rigged configurations, Littewood-Richardson tableaux, catabolizable tableaux

\section{Introduction}

Consider the Levi (block diagonal) subgroup $G L(\eta) \subset G L(n, \mathbb{C})$

$$
G L(\eta)=\prod_{i=1}^{t} G L\left(\eta_{i}, \mathbb{C}\right)
$$

where $\eta=\left(\eta_{1}, \eta_{2}, \ldots, \eta_{t}\right)$ is a sequence of positive integers summing to $n$. Define the Littlewood-Richardson (LR) coefficient to be the multiplicity

$$
L R_{R}^{\lambda}=\operatorname{dim} \operatorname{Hom}_{G L(\eta)}\left(\operatorname{Res}_{G L(\eta)}^{G L(n)} V_{\lambda}, V_{R_{1}} \otimes V_{R_{2}} \otimes \cdots \otimes V_{R_{t}}\right)
$$

where $V_{\lambda}$ is the irreducible $G L(n, \mathbb{C})$ module of highest weight $\lambda$ and $V_{R_{i}}$ is the irreducible $G L\left(\eta_{i}, \mathbb{C}\right)$-module of highest weight $R_{i}$. There is a well-known set $\operatorname{LRT}(\lambda ; R)$ of Young tableaux (which shall be referred to as LR tableaux) whose cardinality is the above coefficient $L R_{R}^{\lambda}[22]$.

In [41] one of the authors and J. Weyman began the combinatorial study of a family of polynomials $K_{\lambda ; R}(q)$ which are $q$-analogues of the LR coefficients $L R_{R}^{\lambda}$ and are by definition the Poincaré polynomials of isotypic components of Euler characteristics of certain $\mathbb{C}\left[\mathrm{gl}_{n}\right]$-modules supported in nilpotent conjugacy class closures. The polynomials $K_{\lambda ; R}(q)$ were conjecturally described as the generating function over catabolizable tableaux with the charge statistic, giving a simultaneous generalization of two formulas of Lascoux 
and Schützenberger for the Kostka-Foulkes polynomials [19, 23]. The Kostka-Foulkes polynomials occur as special cases in two different ways, namely, when each partition $R_{i}$ is a single row, or each is a single column. When $\mu$ has two columns, the Macdonald-Kostka polynomial $K_{\lambda, \mu}(q, t)$ has a nice formula in terms of the polynomials $K_{\lambda ; R}(q)$ where each partition $R_{i}$ has size at most $2[7,40]$.

Our point of departure is the observation that when each $R_{i}$ is a rectangular partition, the polynomial $K_{\lambda ; R}(q)$ seems to coincide with another $q$-analogue of the appropriate LR coefficients, given by the set $R C(\lambda ; R)$ of rigged configurations [18]. One of the authors had already given a bijection $\Psi_{R}: \operatorname{LRT}(\lambda ; R) \rightarrow R C(\lambda ; R)$ [10]. The latter set is endowed with a natural statistic $R C(\lambda ; R) \rightarrow \mathbb{N}$. An obvious problem is to give a direct description of the statistic on $\operatorname{LRT}(\lambda ; R)$ that is obtained by pulling back the statistic on $R C(\lambda ; R)$ via the bijection $\Psi_{R}$. We offer two conjectures for this statistic, which generalize the formulas for the charge statistic given by Donin [5] and Lascoux, Leclerc, and Thibon [20].

Like the LR coefficients of which they are $q$-analogues, the polynomials $K_{\lambda ; R}(q)$ satisfy symmetry and monotonicity properties that extend those satisfied by the Kostka-Foulkes polynomials [41]. Indeed, some of these symmetries only appear after generalizing from the Kostka-Foulkes case to the rectangular LR case. We give bijections and injections that exhibit these properties combinatorially, for each of the three kinds of objects (LR tableaux, catabolizable tableaux, and rigged configurations). In particular, the monotonicity property is exhibited by functorial statistic-preserving embeddings of families of LR tableaux, generalizing the theory of the cyclage due to Lascoux and Schützenberger [19, 25].

There is another $q$-analogue of LR coefficients introduced by Lascoux, Leclerc, and Thibon $[15,20]$. These polynomials arise in a completely different manner, namely, as coefficient polynomials in a generating function over ribbon tableaux. We conjecture that the polynomials $K_{\lambda ; R}(q)$ coincide with theirs.

The paper is organized as follows. Section 2 recalls the definition of the polynomial $K_{\lambda ; R}(q)$ and its symmetry and monotonicity properties. Sections 3 through 5 give the three conjectured combinatorial descriptions for the polynomials $K_{\lambda ; R}(q)$. Section 6 gives (conjecturally statistic-preserving) bijections $\Psi_{R}$ from LR tableaux to rigged configurations and $\Psi_{\text {rows }(R)}$ from rigged configurations to catabolizable tableaux. For each of these kinds of objects Sections 7 through 10 give bijections and injections that reflect the symmetry and monotonicity properties of the polynomials $K_{\lambda ; R}(q)$. These maps were defined so that they are intertwined by the maps $\Psi_{R}$ and $\Psi_{\text {rows }(R)}$.

\subsection{Update}

The original version of this manuscript was submitted for publication in 1998. Three years passed before it was refereed. During that time many of the conjectures became theorems. The text is unchanged except for corrections and minor points of exposition. In this section we shall indicate which conjectures have been proven, and where the proofs may be found.

There are several areas where the so-called generalized Kostka polynomials have arisen (see the survey paper [12]). We give names to the polynomials which are naturally defined in these various contexts. 
1. Twisted modules supported on the nullcone [37, 38, 41]. $K_{\lambda ; R}(q)$ (defined in (2.2)) is the graded Euler characteristic character of such a module. In [41] the notion of a catabolizable tableau is introduced, and $C T_{\lambda ; R}(q)$ is defined in (5.1) as the generating function of such tableaux by the charge statistic.

2. Cyclage theory of Littlewood-Richardson tableaux, developed independently in 1998 by Schilling and Warnaar [35] and the second author [37, 38]. $L R T_{\lambda ; R}(q)$ is defined in (4.3) as the generating function of Littlewood-Richardson tableaux, except that the statistic (7.1) should used instead of (4.2).

3. Affine crystal theory $[35,39]$. Here we shall denote by $X_{\lambda ; R}(q)$ the classically restricted one-dimensional sum, defined, for example, in [35, Def. 3.8].

4. Multiplicities of Bethe vectors $[16,18,35] . R C_{\lambda ; R}(q)$ is defined in (3.4) as the generating function over rigged configurations. See also the survey article [13].

5. Parabolic affine Kazhdan-Lusztig polynomials $[20,26]$. We shall denote these by $c_{R}^{\lambda}(q)$; it is denoted $\tilde{K}_{\Lambda \lambda}^{(p)}(q)$ in Section 2.10, where $p$ and $\Lambda$ are determined by $R$.

$K=L R T$ is proved in [37, Thm. 10]. Up to the (unproven) equality of the two charge ${ }_{R}$ statistics (4.2) and (7.1), this is Conjecture 7. $L R T=X$ is proved in [35, Cor. 5.2] and [39]. $L R T=R C$ follows from [16, Def.-Prop. 4.1, Thm. 9.1]. The equality $L R T=C T$ is proved in the case that each rectangle is contained in the previous one, by [38, Thms. 4, 10]; perhaps the closest result to proving this equality is [38, Thm. 21]. The equality $L R T=c$ (Conjecture 5 ) is the most important conjecture in this paper that remains open.

We now proceed through the rest of the conjectures in order. Conjectures 2 and 3 (for $L R T$ ) follow from [35, Thm. 7.1] and [38, Thm. 30]. Conjecture 4 is true for $K$ using an easy geometric argument; see the discussion of [38, Thm. 2]. For $L R T$ it is an interesting combinatorial problem, settled by [35, (6.11)] and [38, Thm. 4]. Conjecture 6 follows from $K=L R T=R C$. Conjecture 8 is known when $L R T=C T$ is (see the previous paragraph). We believe Conjecture 8 holds for any dominant sequence of partitions $R$, not only for rectangles. Conjecture 9 is [16, Thm. 9.1]. Conjecture 10 is equivalent to Conjecture 8 in light of Conjecture 18, which is proved in [16, Thm. 8.3]. Conjecture 11 is [16, Lemma 8.5]. Conjecture 12 is [16, Thm. 5.6]. Conjecture 13 follows from [38, Thm. 4] and Conjecture 18 , except for the statement about catabolizable tableaux. Conjecture 14 is [30, Thm. 5.7]. Conjecture 15 can be shown to hold using the results of [38] in the case that each rectangle is contained in the previous one. Conjecture 16 is [16, Thm. 7.1]. Conjecture 17 follows from results in [38, Sec. 3].

\section{Definition and properties of $K_{\lambda ; R}(q)$}

The material in this section essentially follows [41].

\subsection{Generating function definition}

Let $\eta=\left(\eta_{1}, \eta_{2}, \ldots, \eta_{t}\right)$ be a sequence of positive integers that sum to $n, \gamma=\left(\gamma_{1}, \gamma_{2}, \ldots, \gamma_{n}\right)$ an integral weight (that is, $\gamma \in \mathbb{Z}^{n}$ ), and $\operatorname{Roots}_{\eta}$ the set of ordered pairs $(i, j)$ such that 
$1 \leq i \leq \eta_{1}+\eta_{2}+\cdots+\eta_{r}<j \leq n$ for some $r$. Let $\Sigma_{A}$ be the symmetric group on the set $A,[a, b]$ the closed interval of integers $i$ with $a \leq i \leq b,[n]=[1, n], x=\left(x_{1}, x_{2}, \ldots, x_{n}\right)$ a sequence of variables, $x^{\gamma}=x_{1}^{\gamma_{1}} x_{2}^{\gamma_{2}} \cdots x_{n}^{\gamma_{n}}$, and $\rho=(n-1, n-2, \ldots, 1,0)$. The symmetric group $\Sigma_{[n]}$ acts on polynomials in $x$ by permuting variables. Define the operators $J$ and $\pi$ by

$$
\begin{aligned}
J(f) & =\sum_{w \in \Sigma_{[n]}}(-1)^{w} w\left(x^{\rho} f\right) \\
\pi f & =J(1)^{-1} J(f)
\end{aligned}
$$

$J(1)$ is the Vandermonde determinant. For the dominant (weakly decreasing) integral weight $\lambda=\left(\lambda_{1} \geq \lambda_{2} \geq \cdots \geq \lambda_{n}\right)$, the character $s_{\lambda}(x)$ of $V_{\lambda}$ is given by the Laurent polynomial

$$
s_{\lambda}(x)=\pi x^{\lambda} .
$$

When $\lambda$ is a partition (that is, $\lambda_{n} \geq 0$ ), $s_{\lambda}$ is the Schur polynomial.

Let $B_{\eta}(x ; q), H_{\gamma, \eta}(q)$, and $K_{\lambda, \gamma, \eta}(q)$ be the formal power series defined by

$$
\begin{aligned}
B_{\eta}(x ; q) & =\prod_{(i, j) \in \operatorname{Roots}_{\eta}} \frac{1}{1-q x_{i} / x_{j}} \\
H_{\gamma, \eta}(x ; q) & =\pi\left(x^{\gamma} B_{\eta}(x ; q)\right) \\
& =\sum_{\lambda} s_{\lambda}(x) K_{\lambda, \gamma, \eta}(q),
\end{aligned}
$$

where $\lambda$ runs over the dominant integral weights in $\mathbb{Z}^{n}$. Ostensibly given by power series, the $K_{\lambda, \gamma, \eta}(q)$ are in fact polynomials with integer coefficients [41].

Let $R$ be the sequence $\left(R_{1}, R_{2}, \ldots, R_{t}\right)$ with $R_{i} \in \mathbb{Z}^{\eta_{i}}$ a dominant integral weight for all $i$, and $\gamma(R) \in \mathbb{Z}^{n}$ the weight obtained by concatenating the parts of the $R_{i}$ in order. Define

$$
K_{\lambda ; R}(q):=K_{\lambda, \gamma(R), \eta}(q) .
$$

It is known [41] that

$$
K_{\lambda ; R}(1)=L R_{R}^{\lambda}
$$

From now on it is assumed that $\lambda$ is a partition and each $R_{i}$ is a rectangular partition having $\eta_{i}$ rows and $\mu_{i}$ columns.

\subsection{Special cases}

Let $\lambda$ be a partition.

1. Let $R_{i}$ be the single row $\left(\mu_{i}\right)$ for all $i$, where $\mu$ is a partition of length at most $n$. Then

$$
K_{\lambda ; R}(q)=K_{\lambda, \mu}(q)
$$


the Kostka-Foulkes polynomial. For a definition of the Kostka-Foulkes polynomials (as well as the cocharge and Macdonald-Kostka versions mentioned below) see [27].

2. Let $R_{i}$ be the single column $\left(1^{\eta_{i}}\right)$ for all $i$. Then

$$
K_{\lambda ; R}(q)=\tilde{K}_{\lambda^{t}, \eta^{+}}(q),
$$

the cocharge Kostka-Foulkes polynomial, where $\lambda^{t}$ is the transpose or conjugate of the partition $\lambda$ and $\eta^{+}$is the partition obtained by sorting the parts of $\eta$ into weakly decreasing order.

3. Let $k$ be a positive integer and $R_{i}$ the rectangle with $k$ columns and $\eta_{i}$ rows. Then $K_{\lambda ; R}(q)$ is the Poincare polynomial of the isotypic component of the irreducible $G L(n)$-module of highest weight $\left(\lambda_{1}-k, \lambda_{2}-k, \ldots, \lambda_{n}-k\right)$ in the coordinate ring of the Zariski closure of the nilpotent conjugacy class whose Jordan form has diagonal block sizes given by the transpose of the partition $\eta^{+}$. In the case $\mu=\left(1^{n}\right)$ these are Kostant's generalized exponents in type $A$.

4. Let $\lambda$ be a partition of $n$ and $\mu$ a two-column partition. In this case J. Stembridge [40] gave an explicit formula for the Macdonald-Kostka polynomials, which has the form

$$
K_{\lambda,\left(2^{r}, 1^{n-2 r}\right)}(q, t)=\sum_{k=0}^{r} q^{k}\left[\begin{array}{l}
r \\
k
\end{array}\right]_{t} M_{r-k}^{k}(t)
$$

where the $M_{r-k}^{k}(t)$ are members of a family of polynomials $M_{m}^{d}(t)$ that are defined by iterated degree-shifted differences of ordinary Kostka-Foulkes polynomials. S. Fishel [7] gave a combinatorial description of the polynomials $M_{m}^{d}(t)$ in terms of rigged configurations, using a variation of the original statistic of [18] on the set of rigged configurations corresponding to standard tableaux. Using the original statistic but replacing standard tableaux by tableaux corresponding to sequences of tiny rectangles of the form $(2),(1,1)$, and (1), we have

$$
M_{r-k}^{k}(t)=K_{\lambda,\left((2)^{r-k},(1,1)^{k},(1)^{n-2 r}\right)}(t) .
$$

The polynomials $M_{m}^{d}(t)$ are defined by a recurrence that may be interpreted in terms of minimal degenerations of nilpotent conjugacy class closures [14]. If the right hand side of (2.4) is replaced by a conjecturally equivalent formulation involving rigged configurations, the resulting formula is proven in Section 10.

\subsection{Defining recurrence}

The polynomials $K_{\lambda ; R}(q)$ satisfy the following recurrence which generalizes Morris' recurrence for the Kostka-Foulkes polynomials [29] and Weyman's recurrence for the Poincaré polynomials in case (3) above [43]. The polynomials $K_{\lambda ; R}(q)$ are uniquely defined by the initial condition

$$
K_{\lambda ;\left(R_{1}\right)}(q)=\delta_{\lambda, R_{1}}
$$


and the recurrence

$$
K_{\lambda ; R}(q)=\sum_{w \in \Sigma_{[n]} / \Sigma_{\left[\eta_{1}\right]} \times \Sigma_{\left[\eta_{1}+1, n\right]}}(-1)^{w} q^{|\alpha(w)|} \sum_{\tau} K_{\tau, \hat{R}}(q) L R_{\alpha(w), \beta(w)}^{\tau}
$$

where $w$ runs over the minimal length permutation in each coset in $\Sigma_{[n]}$ of the given Young subgroup, $\alpha(w)$ and $\beta(w)$ are the first $\eta_{1}$ and last $n-\eta_{1}$ parts of the weight $w^{-1}(\lambda+\rho)-$ $\left(R_{1}+\rho\right)$, and $\hat{R}=\left(R_{2}, R_{3}, \ldots, R_{t}\right)$. The $w$-th summand is understood to be zero if $\alpha(w)$ has a negative part.

The case that $R=\left(R_{1}, R_{2}\right)$ is now calculated explicitly. Suppose $\mu_{1} \geq \mu_{2}$. It is easy to show that for any $\lambda$,

$$
L R_{\left(R_{1}, R_{2}\right)}^{\lambda} \in\{0,1\}
$$

Assuming this multiplicity is one, it follows from (2.5) that

$$
K_{\lambda ;\left(R_{1}, R_{2}\right)}(q)=q^{d}
$$

where $d$ is the number of cells in $\lambda$ strictly to the right of the $\mu_{1}$-th column.

Example 1 We give a running example. Let $n=9, \mu=(3,2,1), \eta=(2,4,3)$. Let

$$
R=((3,3),(2,2,2,2),(1,1,1)) \quad \lambda=(5,4,3,2,2,1,0,0,0)
$$

Applying (2.5), all summands are zero except for the identity permutation. Using the LR rule, it is not hard to see that the summand for $\tau$ is zero unless $\tau$ is one of the three partitions $(3,3,3,2),(3,3,2,2,1)$, and $(3,2,2,2,1,1)$. Three applications of $(2.7)$ yield

$$
\begin{aligned}
K_{\lambda ; R}(q) & =q^{3}\left(K_{(3,3,3,2), \hat{R}}(q)+2 K_{(3,3,2,2,1), \hat{R}}(q)+K_{(3,2,2,2,1,1), \hat{R}}(q)\right) \\
& =q^{3}\left(q^{3}+2 q^{2}+q^{1}\right)=q^{6}+2 q^{5}+q^{4} .
\end{aligned}
$$

\subsection{Positivity}

The sequence $R$ is said to be dominant if $\gamma(R)$ is. The following positivity conjecture and theorem are due to Broer.

Conjecture 1 ([2]) If $R$ is dominant (but not necessarily a sequence of rectangles) then $K_{\lambda ; R}(q) \in \mathbb{N}[q]$.

Theorem 1 ([1]) Conjecture 1 holds when $R$ is a sequence of rectangles.

Example 2 For $\lambda=(2,2)$ and the non-dominant sequence of rectangles $R=\{(1),(3)\}$, $K_{\lambda ; R}(q)=q-1$. 


\subsection{Cocharge normalization}

Given a sequence of rectangles $R$, let $r_{i, j}(R)$ be the number of rectangles in $R$ that contain the cell $(i, j)$, or equivalently, the number of indices $a$ such that $\mu_{a} \geq i$ and $\eta_{a} \geq j$. Define the number

$$
n(R)=\sum_{(i, j)}\left(\begin{array}{c}
r_{i, j}(R) \\
2
\end{array}\right)
$$

and the polynomial

$$
\tilde{K}_{\lambda ; R}(q)=q^{n(R)} K_{\lambda ; R}\left(q^{-1}\right)
$$

When $R$ consists of single-rowed shapes, the above assertion and both the two KostkaFoulkes special cases imply that

$$
K_{\lambda, \mu}(q)=q^{n(\mu)} \tilde{K}_{\lambda, \mu}\left(q^{-1}\right)
$$

where $n(\mu)=\sum_{j}\left(\begin{array}{c}\mu_{j}^{t} \\ 2\end{array}\right)$. This coincides with the definition of the cocharge Kostka-Foulkes polynomials.

Example 3 In our running example, the matrix $\left(r_{i, j}\right)$ is given by

$$
\left(r_{i, j}\right)=\begin{array}{lllll}
3 & 2 & 1 & 0 & \ldots \\
3 & 2 & 1 & 0 & \ldots \\
2 & 1 & 0 & 0 & \ldots \\
1 & 1 & 0 & 0 & \ldots
\end{array}
$$

with all other entries zero, so $n(R)=2\left(\begin{array}{l}3 \\ 2\end{array}\right)+3\left(\begin{array}{l}2 \\ 2\end{array}\right)=9$ and $\tilde{K}_{\lambda ; R}(q)=q^{5}+2 q^{4}+q^{3}$.

Here is another version of the positivity conjecture, which adds an observation about an upper bound on the powers of $q$ that may occur.

Conjecture 2 For $R$ dominant, $\tilde{K}_{\lambda ; R}(q) \in \mathbb{N}[q]$.

\subsection{Reordering symmetry}

Theorem 2 ([41]) Suppose $R$ and $R^{\prime}$ are dominant sequences of rectangles that are rearrangements of each other. Then $K_{\lambda ; R}(q)=K_{\lambda ; R^{\prime}}(q)$.

This property is not obvious since the reordering of the sequence of rectangles results in a major change in the generating function $H_{\gamma, \eta}(x ; q)$. 


\subsection{Contragredient duality symmetry}

Let $\operatorname{rev}(\eta)$ denote the reverse of $\eta$. Fix a positive integer $k$ such that $k \geq \lambda_{1}$ and $k \geq \mu_{i}$ for all $i$. Let $\tilde{\lambda}=\left(k-\lambda_{n}, k-\lambda_{n-1}, \ldots, k-\lambda_{1}\right)$ and $\tilde{R}_{i}=\left(\left(k-\mu_{i}\right)^{\eta_{i}}\right)$ for $1 \leq i \leq t$. Note that $\tilde{\lambda}$ (resp. $\tilde{R}_{i}$ ) is obtained by the 180 degree rotation of the complement of the partition $\lambda$ (resp. $R_{i}$ ) inside the $k \times n$ rectangle (resp. $k \times \eta_{i}$ rectangle). Then

Proposition 3 ([41]) For R dominant,

$$
K_{\lambda ; R}(q)=K_{\tilde{\lambda} ; \operatorname{rev}(\tilde{R})}(q) .
$$

Example 4 In our running example, take $k=5$. Then we have

$$
K_{(5,4,3,2,2,1,0,0,0),((3,3),(2,2,2,2),(1,1,1))}(q)=K_{(5,5,5,4,3,3,2,1,0),((4,4,4),(3,3,3,3),(2,2))}(q) .
$$

\subsection{Transpose symmetry}

Let $R^{t}$ be the sequence of rectangles obtained by transposing each of the rectangles in $R$.

Conjecture 3 Let $R$ be dominant and $R^{\prime}$ a dominant rearrangement of $R^{t}$. Then

$$
K_{\lambda^{t} ; R^{\prime}}(q)=\tilde{K}_{\lambda ; R}(q),
$$

where the left hand side is computed in $G L(m)$ where $m$ is the total number of columns in the rectangles of $R$.

This property is mysterious; it is not obvious from the properties of the modules that define the polynomials $K_{\lambda ; R}(q)$.

Example 5 In the running example, $\lambda^{t}=(6,5,3,2,1,0)$ and

$$
\begin{aligned}
& R^{t}=((2,2,2),(4,4),(3)) \\
& R^{\prime}=((4,4),(3),(2,2,2))
\end{aligned}
$$

Using (2.5) we have

$$
K_{(6,5,3,2,1,0),((4,4),(3),(2,2,2))}(q)=q^{5}+2 q^{4}+q^{3},
$$

which agrees with the computation of $\tilde{K}_{\lambda ; R}(q)$ in Example 3.

\subsection{Monotonicity}

Let $\alpha$ and $\beta$ be sequences of nonnegative integers. Say that $\alpha \unrhd \beta$ if $\alpha^{+} \unrhd \beta^{+}$in the dominance partial order on partitions. 
Given a sequence of rectangles $R$, let $\tau^{k}(R)$ be the partition whose parts consist of the $\eta_{i}$ such that $\mu_{i}=k$, sorted into decreasing order. In other words, $\tau^{k}(R)$ is the multiset of heights of the rectangles in $R$ that have exactly $k$ columns. Say that $R \unrhd R^{\prime}$ if $\tau^{k}(R) \unrhd \tau^{k}\left(R^{\prime}\right)$ for all $k$.

Conjecture 4 Suppose $R$ and $R^{\prime}$ are dominant with $R \unrhd R^{\prime}$. Then $K_{\lambda ; R^{\prime}}(q) \geq K_{\lambda ; R}(q)$ coefficientwise.

Example 6 Let $R$ be as usual and $R^{\prime}=((3),(3),(2,2,2,2),(1,1,1))$. The sequence of partitions $\tau^{k}(R)$ and $\tau^{k}\left(R^{\prime}\right)$ are given by

$$
\begin{aligned}
\tau \cdot(R) & =((3),(4),(2),(), \ldots) \\
\tau \cdot\left(R^{\prime}\right) & =((3),(4),(1,1),(), \ldots)
\end{aligned}
$$

Now

$$
K_{\lambda ; R^{\prime}}(q)=2 q^{7}+4 q^{6}+3 q^{5}+q^{4}
$$

which dominates $K_{\lambda ; R}(q)$ coefficientwise.

Suppose all of the rectangles have the same number of columns $k$, so that $\tau^{j}$ is empty for $j \neq k$. In this case Conjecture 4 may be verified as follows. For the partition $\mu$ of $n$, let $A_{\mu}$ be the coordinate ring of the closure of the nilpotent conjugacy class of matrices with transpose Jordan type $\mu$. If $v$ is another partition with $\mu \unrhd v$, then restriction of functions gives a natural epimorphism of graded $G L(n)$-modules $A_{v} \rightarrow A_{\mu}$. This special case includes a monotonicity property of the cocharge Kostka polynomials:

$$
\tilde{K}_{\lambda, v}(q) \geq \tilde{K}_{\lambda, \mu}(q) \quad \text { if } \mu \unrhd v .
$$

The first combinatorial proof of this fact was given in [19, 24, 25]. It uses two substantial results. The first is the original interpretation [23] of the Kostka-Foulkes polynomial $K_{\lambda, \mu}(q)$ as the generating function over the set $T(\lambda, \mu)$ of tableaux of shape $\lambda$ and content $\mu$ with the charge statistic. The second is a cocharge-preserving embedding of $T(\lambda, \mu)$ into $T(\lambda, v)$. An alternate proof of this fact is given in $[3,11]$. Under the statistic-preserving bijection in [18] that sends tableaux to rigged configurations, the image $T(\lambda, \mu)$ is easily seen to be a subset in the image of $T(\lambda, v)$, yielding (2.12).

\subsection{Generalized Kostka polynomials and ribbon tableaux}

According to (2.3) and Conjecture 2, the generalized Kostka polynomials are $q$-analogues of tensor product multiplicities. Another $q$-analogue of tensor product multiplicities was introduced by A. Lascoux, B. Leclerc and J.-Y. Thibon [20] using the spin generating functions for the set of $p$-ribbon tableaux. We refer the reader to [20, Sections 4 and 6] for the definitions of the notions of a $p$-ribbon tableau $T$, the spin $s(T)$ of a $p$-ribbon tableau, 
and the " $p$-ribbon version" of the modified Hall-Littlewood polynomials $\tilde{G}_{\Lambda}^{(p)}\left(X_{n} ; q\right)$. Let $\Lambda$ be the partition with empty $p$-core and $p$-quotient $\left(R_{1}, R_{2}, \ldots, R_{p}\right)$ (see $[27$, Chapter 1 , Example 8], for the definitions of the $p$-core and $p$-quotient of a partition $\lambda$ ). By definition

$$
\tilde{G}_{\Lambda}^{(p)}\left(X_{n} ; q\right)=\sum_{T \in \operatorname{Tab}_{p}(\Lambda, \leq n)} q^{\tilde{s}(T)} x^{w t(T)},
$$

where the sum runs over the $\operatorname{set} \operatorname{Tab}_{p}(\Lambda, \leq n)$ of $p$-ribbon tableaux of shape $\Lambda$ filled with numbers not exceeding $n, w t(T)$ is the weight or content of the ribbon tableau $T$, and $\tilde{s}(T)=-s(T)+\max \left\{s(T) \mid T \in \operatorname{Tab}_{p}(\Lambda, \leq n)\right\}$ is the cospin of the $p$-ribbon tableau $T$, cf. [20, (25)]. It is known [20, Theorem 6.1] that $\tilde{G}_{\Lambda}^{(p)}\left(X_{n} ; q\right)$ is a symmetric function. Following [20], let us define polynomials $\tilde{K}_{\Lambda \lambda}^{(p)}(q)$ via the decomposition

$$
\tilde{G}_{\Lambda}^{(p)}\left(X_{n} ; q\right)=\sum_{\Lambda} \tilde{K}_{\Lambda \lambda}^{(p)}(q) s_{\lambda}\left(X_{n}\right)
$$

The $p$-quotient bijection of Littlewood, induces a weight-preserving bijection from $p$-ribbon tableaux of shape $\Lambda$ to $p$-tuples of tableaux of shapes $R_{1}, R_{2}, \ldots, R_{p}$ [42], yielding the equality

$$
\tilde{G}_{\Lambda}^{(p)}\left(X_{n} ; 1\right)=s_{R_{1}}\left(X_{n}\right) s_{R_{2}}\left(X_{n}\right) \cdots s_{R_{p}}\left(X_{n}\right)
$$

Taking the coefficient of $s_{\lambda}\left(X_{n}\right)$, it follows that $\tilde{G}_{\Lambda}^{(p)}\left(X_{n} ; 1\right)$ is the multiplicity of the highest weight $s l(n)$-module $V_{\lambda}$ in the tensor product $V_{R_{1}} \otimes \cdots \otimes V_{R_{p}}$.

Recall that a sequence of partitions $R=\left(R_{1}, \ldots, R_{p}\right)$ is called dominant, if for all $1 \leq i \leq p-1$, the last part of $R_{i}$ is at least as large as the first part of $R_{i+1}$.

Conjecture 5 Let $R=\left(R_{1}, \ldots, R_{p}\right)$ be a dominant sequence of rectangular partitions, and $\Lambda$ the partition with empty $p$-core and $p$-quotient $\left(R_{1}, \ldots, R_{p}\right)$. Then

$$
\tilde{K}_{\lambda ; R}(q)=\tilde{K}_{\Lambda \lambda}^{(p)}(q) .
$$

\section{Rigged configurations}

Rigged configurations are combinatorial objects that provide a new interpretation of the Kostka-Foulkes polynomial [18]. However, this construction applies for arbitrary sequences of rectangles, not just those consisting of all single-rowed rectangles. This section follows [18].

Let $\lambda$ be a partition and $R$ a sequence of rectangular partitions such that $|\lambda|=\sum_{i}\left|R_{i}\right|$. Let $R_{i}$ have $\eta_{i}$ rows and $\mu_{i}$ columns as usual. A configuration of type $(\lambda ; R)$ is a sequence of partitions $v=\left(v^{1}, v^{2}, \ldots\right)$ such that

$$
\begin{aligned}
\left|v^{k}\right| & =\sum_{j>k} \lambda_{j}-\sum_{a \geq 1} \mu_{a} \max \left(\eta_{a}-k, 0\right) \\
& =-\sum_{j \leq k} \lambda_{j}+\sum_{a \geq 1} \mu_{a} \min \left(k, \eta_{a}\right)
\end{aligned}
$$


for each $k \geq 1$. Note that if $k \geq \ell(\lambda)$ and $k \geq \eta_{a}$ for all $a$, then $v^{k}$ is empty. We make the convention that $v^{0}$ is the empty partition.

For a partition $\rho$, let $Q_{n}(\rho)=\rho_{1}^{t}+\rho_{2}^{t}+\cdots+\rho_{n}^{t}$ be the number of cells in the first $n$ columns of $\rho$. The vacancy numbers of the configuration $v$ of type $(\lambda ; R)$ are defined by

$$
P_{k, n}(v)=Q_{n}\left(v^{k-1}\right)-2 Q_{n}\left(v^{k}\right)+Q_{n}\left(v^{k+1}\right)+\sum_{a \geq 1} \min \left(\mu_{a}, n\right) \delta_{\eta_{a}, k}
$$

for $k, n \geq 1$, where $\delta_{i, j}$ is the Kronecker delta. Say that a configuration $v$ of type $(\lambda ; R)$ is admissible if $P_{k, n}(v) \geq 0$ for all $k, n \geq 1$.

Example 7 In the running example, there is a unique admissible configuration of type $(\lambda ; R)$, given by

$$
v=((1),(2,1),(2,1),(2,1),(1))
$$

Below is the table $P_{k, n}(v)$ of vacancy numbers for $k, n \geq 1$ with $k$ as row index and $n$ as column index.

$$
\begin{array}{cccc}
0 & 1 & 1 & \ldots \\
0 & 0 & 1 & \ldots \\
1 & 1 & 1 & \ldots \\
0 & 0 & 0 & \ldots \\
0 & 1 & 1 & \ldots \\
1 & 1 & 1 & \ldots \\
0 & 0 & 0 & \ldots \\
\vdots & \vdots & \vdots & \ldots
\end{array}
$$

A rigging $L$ of an admissible configuration $v$ of type $(\lambda ; R)$ consists of an integer label $L_{s}^{k}$ for each row $v_{s}^{k}$ of each of the partitions of $v$, such that:

1. $0 \leq L_{s}^{k} \leq P_{k, v_{s}^{k}}(v)$ for every $k \geq 1$ and $1 \leq s \leq \ell\left(v^{k}\right)$.

2. If $v_{s}^{k}=v_{s+1}^{k}$, then $L_{s}^{k} \geq L_{s+1}^{k}$.

Only some of the vacancy numbers $P_{k, n}(v)$ appear as upper bounds for the labels $L_{s}^{k}$, namely, those where $n$ is a part of the partition $v^{k}$. The second condition merely says that the labels for a fixed partition $v^{k}$ and part size $n$, should be viewed as a multiset.

Example 8 To indicate the maximum rigging $L_{\max }$ of the configuration $v$, we replace each part $v_{s}^{k}$ by its maximum label $P_{k, \nu_{s}^{k}}(\nu)$, obtaining

$$
L_{\max }=((0),(0,0),(1,1),(0,0),(0))
$$


So $v$ has the four riggings

$$
\begin{array}{ll}
((0),(0,0),(0,0),(0,0),(0)) & ((0),(0,0),(0,1),(0,0),(0)) \\
((0),(0,0),(1,0),(0,0),(0)) & ((0),(0,0),(1,1),(0,0),(0))
\end{array}
$$

A rigged configuration of type $(\lambda ; R)$ is a pair $(\nu, L)$ where $v$ is an admissible configuration of type $(\lambda ; R)$ together with a rigging $L$. Let $C(\lambda ; R)$ denote the set of admissible configurations of type $(\lambda ; R)$ and $R C(\lambda ; R)$ the set of rigged configurations of type $(\lambda ; R)$.

The first important property of rigged configurations is that they are enumerated by the LR coefficient $L R_{R}^{\lambda}$.

Theorem 4 ([18])

$$
L R_{R}^{\lambda}=|R C(\lambda ; R)|=\sum_{\nu \in C(\lambda ; R)} \prod_{k, n \geq 1}\left(\begin{array}{c}
P_{k, n}(v)+m_{n}\left(v^{k}\right) \\
m_{n}\left(v^{k}\right)
\end{array}\right)
$$

where $m_{n}(\rho)$ denotes the number of parts of the partition $\rho$ of size $n$.

Rigged configurations are endowed with a natural statistic we call cocharge. Let $(v, L) \in$ $R C(\lambda ; R)$. Denote by $\alpha_{k, n}=\left(v^{k}\right)_{n}^{t}$ the size of the $n$-th column of the $k$-th partition $v^{k}$ of $v$. The cocharge of $(\nu, L)$ is defined by

$$
\begin{aligned}
\operatorname{cocharge}(v, L) & =\operatorname{cocharge}(v)+\sum_{k \geq 1} \sum_{s=1}^{\ell\left(v^{k}\right)} L_{s}^{k} \\
\operatorname{cocharge}(v) & =\sum_{k, n \geq 1} \alpha_{k, n}\left(\alpha_{k, n}-\alpha_{k+1, n}\right)
\end{aligned}
$$

The $q$-binomial coefficient is defined by

$$
\left(\begin{array}{l}
n \\
k
\end{array}\right)_{q}=\frac{\left(1-q^{n}\right)\left(1-q^{n-1}\right) \cdots\left(1-q^{n-k+1}\right)}{\left(1-q^{k}\right)\left(1-q^{k-1}\right) \cdots(1-q)} .
$$

Define the polynomial $R C_{\lambda ; R}(q)$ by

$$
\begin{aligned}
R C_{\lambda ; R}(q) & =\sum_{(v, L) \in R C(\lambda ; R)} q^{\operatorname{cocharge}(v, L)} \\
& =\sum_{\nu \in C(\lambda ; R)} q^{\operatorname{cocharge}(v)} \prod_{k, n \geq 1}\left(\begin{array}{c}
P_{k, n}(v)+m_{n}\left(v^{k}\right) \\
m_{n}\left(v^{k}\right)
\end{array}\right)_{q} .
\end{aligned}
$$

Conjecture 6 For $R$ dominant,

$$
\tilde{K}_{\lambda ; R}(q)=R C_{\lambda ; R}(q)
$$


Example 9 Let $v$ be the sole member of $R C(\lambda ; R)$ as above. We have

$$
\begin{aligned}
\operatorname{cocharge}(v)= & 1(1-2)+2(2-2)+2(2-2)+2(2-1)+1(1-0) \\
& +0(0-1)+1(1-1)+1(1-1)+1(1-0) \\
= & (-1+2+1)+(-1+1)=3,
\end{aligned}
$$

so that $R C_{\lambda ; R}(q)=q^{3}(1+q)(1+q)$, which agrees with $\tilde{K}_{\lambda ; R}(q)$.

\section{Littlewood-Richardson tableaux}

This section discusses a set $\operatorname{LRT}(\lambda ; R)$ of tableaux that has cardinality $L R_{R}^{\lambda}$.

For the definitions of a (semistandard) tableau, skew tableau, and the (row-reading) word of a tableau $T$ (denoted here by word $(T)$ ), see [6]. We shall often identify a (skew) tableau with its row-reading word. Let $P(w)$ denote Schensted's insertion tableau for the word $w$ [34]; it is the unique tableau of partition shape that is equivalent to $w$ under Knuth's degree three relations [17]. A right factor of the word $w$ is any word $v$ such that $w=u v$ for some word $u$. Say that a word $w$ is lattice if the content of every right factor of $w$ is a partition.

We now fix notation for the sequence of rectangles $R$. Let the intervals $A_{1}, A_{2}$, etc., be given by dividing the interval $[n]$ into successive subintervals of sizes $\eta_{1}, \eta_{2}$, etc. Let $K_{i}$ be the rectangular tableau of shape $R_{i}$ whose $j$-th row consists of $\mu_{i}$ copies of the $j$-th largest letter of the interval $A_{i}$ for $1 \leq j \leq \eta_{i}$.

Example 10 Let $\lambda=(5,4,3,2,2,1)$ and $R=\left(R_{1}, R_{2}, R_{3}\right)$ with $R_{1}=(3,3), R_{2}=$ $(2,2,2,2)$ and $R_{3}=(1,1,1) . \mu=(3,2,1), \eta=(2,4,3), \gamma=(3,3,2,2,2,2,1,1,1)$, $A_{1}=[1,2], A_{2}=[3,6], A_{3}=[7,9]$.

$$
K_{1}=\begin{array}{lll}
1 & 1 & 1 \\
2 & 2 & 2
\end{array} \quad K_{2}=\begin{array}{lll}
3 & 3 & 7 \\
4 & 4 \\
5 & 5 & K_{3}= \\
6 & 6 & 9
\end{array}
$$

Say that a word $v$ in the alphabet $[n]$ is $R-L R$ (short for $R$-Littlewood-Richardson) if the restriction $\left.v\right|_{A_{i}}$ of $v$ to the alphabet $A_{i}$, is lattice in the alphabet $A_{i}$ for all $i$, or equivalently, that $P\left(\left.v\right|_{A_{i}}\right)=K_{i}$ for all $i$. Clearly an $R$-LR word must have content $\gamma(R)$. Let $\operatorname{LRT}(\lambda ; R)$ be the set of $R$-LR words which are also tableaux of shape $\lambda$. We call these LR tableaux. Note that if each rectangle of $R$ is a single row, then $\operatorname{LRT}(\lambda ; R)=$ $T(\lambda, \gamma)$. 
Example $11 \operatorname{LRT}(\lambda ; R)$ consists of the following four tableaux.

$\begin{array}{llllllllllllllllllll}1 & 1 & 1 & 3 & 3 & 1 & 1 & 1 & 3 & 3 & 1 & 1 & 1 & 3 & 7 & 1 & 1 & 1 & 3 & 7 \\ 2 & 2 & 2 & 4 & & 2 & 2 & 2 & 7 & & 2 & 2 & 2 & 4 & & 2 & 2 & 2 & 8 & \\ 4 & 5 & 7 & & & 4 & 4 & 8 & & & 3 & 5 & 8 & & & 3 & 4 & 9 & & \\ 5 & 6 & & & & 5 & 5 & & & & 4 & 6 & & & & 4 & 5 & & \\ 6 & 8 & & & & 6 & 6 & & & & 5 & 9 & & & & 5 & 6 & & \\ 9 & & & & & 9 & & & & & 6 & & & & & 6 & & & & \end{array}$

Let $T$ be the first tableau. The skew tableaux $\left.T\right|_{A_{i}}$ are given below.

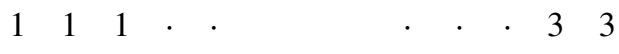

$$
\begin{aligned}
& \begin{array}{lllllll}
2 & 2 & 2 & & & \cdot &
\end{array}
\end{aligned}
$$

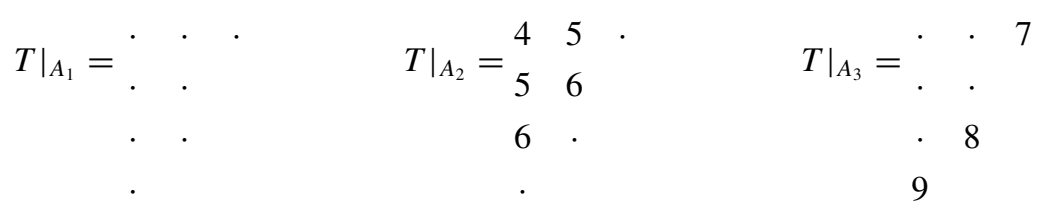

We have $\operatorname{word}\left(\left.T\right|_{A_{1}}\right)=222111, \operatorname{word}\left(\left.T\right|_{A_{2}}\right)=65645433$, and $\operatorname{word}\left(\left.T\right|_{A_{3}}\right)=987$, which are lattice in their respective alphabets.

The classical Littlewood-Richardson rule [22] immediately yields the following result.

Proposition $5 \quad|L R T(\lambda ; R)|=L R_{R}^{\lambda}$

Next we define a statistic charge ${ }_{R}$ on LR tableaux.

Let $v$ be a word of partition content. Say that the sequence of words $\left\{v^{1}, v^{2}, \ldots\right\}$ is a standard decomposition of $v$, if the $v^{i}$ are standard words (words without repeated letters) of weakly decreasing size and $v$ is a shuffle of the words $\left\{v^{i}\right\}$, that is, the $v^{i}$ may be simultaneously disjointly embedded in $v$ and exhaust the letters of $v$.

Now let $R$ be dominant, $v$ an $R$-LR word, and $\left\{v^{i}\right\}$ a standard decomposition of $v$. Say that $\left\{v^{i}\right\}$ is proper if $\left.v^{i}\right|_{A_{j}}$ is either empty or the decreasing word consisting of the letters of $A_{j}$, for all $i$ and $j$. Assuming this holds, let $u^{i}$ be the reverse of the word obtained from $v^{i}$ by replacing each letter in $A_{j}$ by the letter $j$. Define

$$
\operatorname{charge}_{R}(v)=\min _{\left\{v^{i}\right\}} \sum_{i} \operatorname{cocharge}\left(u^{i}\right)
$$

where $\left\{v^{i}\right\}$ runs over the proper standard decompositions of $v$ and $\left\{u^{i}\right\}$ is related to $v^{i}$ as above. Say that a proper standard decomposition of an $R$-LR word $v$ is minimal if it attains the minimum in the definition of charge $_{R}(v)$. 
1. When $\mu$ is a partition and $R_{i}=\left(\mu_{i}\right)$ for all $i$, the subalphabet $A_{j}=\{j\}$, so every standard decomposition is proper and $u^{i}$ is the reverse of $v^{i}$. But for standard words, the cocharge of the reverse of a word equals the charge. So charge ${ }_{R}$ is the usual charge statistic, since Donin [5] asserts that the particular standard decomposition given by Lascoux and Schützenberger [23] is always minimal.

2. When $R_{i}=\left(1^{\eta_{i}}\right)$ for all $i$, an $R$-LR word $v$ is necessarily standard, so $v^{1}=v$ and by definition $\operatorname{charge}_{R}(v)=\operatorname{cocharge}\left(u^{1}\right)$

Example 12 Let $R=((2,2),(2,2,2),(2),(1))$ so that $\mu=(2,2,2,1)$ and $\eta=(2,3$, $1,1)$. The alphabets $A_{j}$ are given by $[1,2],[3,5],[6,6]$ and $[7,7]$. Let $\lambda=(5,4,2,1$, $1,0,0)$. We indicate part of the computation of charge $_{R}$ for the tableau $T$ given below.

$$
\begin{array}{rrrrr}
1 & 1 & 3 & 3 & 6 \\
2 & 2 & 4 & 6 & \\
4 & 5 & & & \\
5 & & & & \\
7 & & & &
\end{array}
$$

We have word $(T)=7.5 .45 .2246 .11336$. It turns out that charge ${ }_{R}(\operatorname{word}(T))=7$. There are at most $2^{3}=8$ distinct proper standard decompositions of word $(T)$ depending on choices involving the letters 4,5 , and 6 ; the pairs of ones, twos, and threes occur side by side and thus do not generate other proper decompositions. The unique minimal decomposition is $v^{1}=7542613, v^{2}=524136$. To see this, reversing these subwords gives $(3162457,631425)$. Replacing each letter in $A_{j}$ by $j$ we have the pair of words $\left(u^{1}, u^{2}\right)=(2131224,321212)$. To take the cocharge we act by automorphisms of conjugation to change them to partition content, obtaining $(2131124,321211)$. Taking the cocharges of these words, we obtain $(3,4)$ whose sum is 7 . Let us now perform the same computation for the circular standard decomposition of Lascoux and Schützenberger (which is always proper): $v^{1}=7524136$ and $v^{2}=542613$. We have $u^{1}=3212124$ and $u^{2}=213122$. Acting by automorphisms of conjugation to move to partition content, we have $(3212114,213112)$, whose cocharges are $(6,2)$ whose sum is 8 , which is not minimal.

It would be desirable to have an algorithm that computes a minimal standard decomposition. The above example shows that the algorithm of Lascoux and Schützenberger for selecting a standard decomposition in the computation of charge, does not work for charge .

For $R$ dominant, define

$$
\operatorname{LRT}_{\lambda ; R}(q)=\sum_{T \in L R T(\lambda ; R)} q^{\operatorname{charge}_{R}(T)}
$$

Conjecture 7 For $R$ dominant,

$$
K_{\lambda ; R}(q)=L R T_{\lambda ; R}(q) .
$$




\section{Catabolizable tableaux}

We recall from [41] the notion of an $R$-catabolizable tableau. Recall the subintervals $A_{i}$ and the canonical rectangular tableaux $K_{i}$ from Section 4. Let $S$ be a tableau of partition shape. Suppose that $\left.S\right|_{A_{1}}=K_{1}$. Let $S_{+}$be the first $\eta_{1}$ rows of the skew tableau $S-K_{1}$ and let $S_{-}$be the remainder. Define the (row) $R_{1}$-catabolism Cat $_{R_{1}}(S)$ of $S$ to be the tableau $P\left(S_{+} S_{-}\right)$. Say that $S$ is $R$-catabolizable if $\left.S\right|_{A_{1}}=K_{1}$ and $\operatorname{Cat}_{R_{1}}(S)$ is $\hat{R}$-catabolizable in the alphabet $\left[\eta_{1}+1, n\right]=[n]-A_{1}$, where $\hat{R}=\left(R_{2}, R_{3}, \ldots\right)$. The empty tableau is considered to be the unique catabolizable tableau for the empty sequence of rectangles. Note that an $R$-catabolizable tableau must have content $\gamma(R)$.

Denote by $C T(\lambda ; R)$ the set of $R$-catabolizable tableaux of shape $\lambda$.

Example 13 Recall the tableaux $K_{1}, K_{2}$, and $K_{3}$ from Example 10. Here are the four tableaux that comprise the set $C T(\lambda ; R)$.

$\begin{array}{llllllllllllllllllll}1 & 1 & 1 & 5 & 6 & 1 & 1 & 1 & 6 & 6 & 1 & 1 & 1 & 5 & 7 & 1 & 1 & 1 & 6 & 7 \\ 2 & 2 & 2 & 6 & & 2 & 2 & 2 & 7 & & 2 & 2 & 2 & 6 & & 2 & 2 & 2 & 8 & \\ 3 & 3 & 7 & & & 3 & 3 & 8 & & & 3 & 3 & 8 & & & 3 & 3 & 9 & \\ 4 & 4 & & & & 4 & 4 & & & & 4 & 4 & & & & 4 & 4 & & \\ 5 & 8 & & & & 5 & 5 & & & & 5 & 9 & & & & 5 & 5 & & \\ 9 & & & & & 9 & & & & & 6 & & & & & 6 & & & \end{array}$

Let $S$ be the first of these tableaux. The following calculation shows that $S$ is $R$ catabolizable.

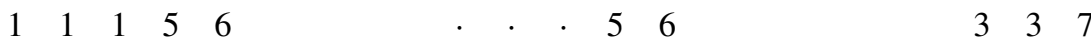

$$
\begin{aligned}
& \begin{array}{llllllllll}
2 & 2 & 2 & 6 & 6 & 6 & 4 & 4
\end{array}
\end{aligned}
$$

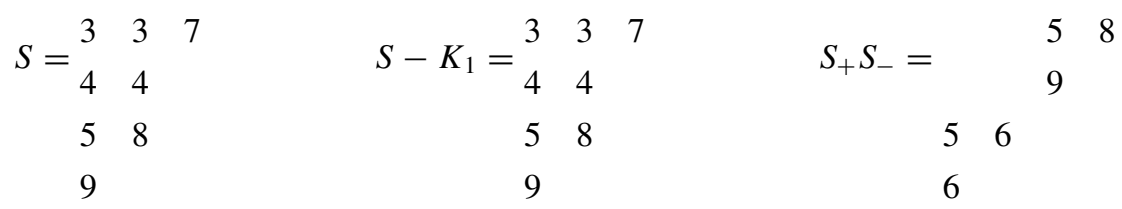

$$
\begin{aligned}
& \mathrm{Cat}_{R_{1}}(S)=\begin{array}{rrr}
3 & 3 & 7 \\
4 & 4 & 8 \\
5 & 5 & 9 \\
6 & 6 &
\end{array} \quad \mathrm{Cat}_{R_{2}} \mathrm{Cat}_{R_{1}} S=\begin{array}{r}
7 \\
8 \\
9
\end{array} \quad \mathrm{Cat}_{R_{3}} \mathrm{Cat}_{R_{2}} \mathrm{Cat}_{R_{1}} S=\emptyset
\end{aligned}
$$

Define

$$
C T_{\lambda ; R}(q)=\sum_{S \in C T(\lambda ; R)} q^{\operatorname{charge}(S)} .
$$


Conjecture 8 Let $R$ be dominant. Then

$$
K_{\lambda ; R}(q)=C T_{\lambda ; R}(q)
$$

When each rectangle in $R$ is a single row, this formula (as well as that in Conjecture 7) specializes to the description of the Kostka-Foulkes polynomials in [23].

When each rectangle is a single column, this formula specializes (with some work [41]) to a formula for the cocharge Kostka-Foulkes polynomials [19].

\section{Bijections between the three sets}

In [18] two bijections $L R T(\lambda ; R) \rightarrow R C(\lambda ; R)$ were given, in the case that all were single rows or all single columns. When all are single rows, $\operatorname{LRT}(\lambda ; R)=T(\lambda, \mu)$, the set of tableaux of shape $\lambda$ and content $\mu$. When all rectangles are single columns, there is an obvious bijection $\operatorname{LRT}(\lambda ; R) \rightarrow T\left(\lambda^{t}, \eta\right)$ given by taking the transpose of a tableau and then relabelling (see the LR-transpose map in Section 9). In [18] it is asserted that the first of these two bijections is statistic-preserving. One of the authors [10] has given a common generalization of these bijections, which we denote by

$$
\Phi_{R}: \operatorname{LRT}(\lambda ; R) \rightarrow R C\left(\lambda^{t} ; R^{t}\right)
$$

For those that have some familiarity with [18] we point out two twists in its definition. The first difference is in the labelling convention. In [18] the bijections use the notion of a singular string in a rigged configuration $(\nu, J)$, that is, a part $\nu_{s}^{k}$ whose label $J_{s}^{k}$ attains the maximum value $P_{k, v_{s}^{k}}(v)$. This convention is called the quantum number labelling. Here we employ the coquantum number labelling, in which a singular string is a part $v_{s}^{k}$ whose label $L_{s}^{k}$ is zero. The second difference is the direction in which the cells of the rectangles $R_{i}$ are ordered. In [18] the cells of the one-rowed rectangles are ordered along rows, but here the cells of the rectangles are ordered along columns. This accounts for the transposing of shapes in passing from LR tableaux to rigged configurations.

Conjecture 9 For $R$ dominant, the bijection $\Phi_{R}$ is statistic-preserving, that is, for $T \in$ $\operatorname{LRT}(\lambda ; R)$ and $(\nu, L)=\Phi_{R}(T)$, we have

$$
\operatorname{charge}_{R}(T)=\operatorname{cocharge}(v, L) \text {. }
$$

The dominance of $R$ is only necessary for the definition of charge . .

This bijection may also be used to define a map from rigged configurations to catabolizable tableaux. Let rows $(R)$ be the sequence of rectangles obtained by slicing each rectangle of $R$ into single rows. Recall the weight $\gamma=\gamma(R)$; its parts give the single-rowed shapes of $\operatorname{rows}(R)$. In our running example, $\operatorname{rows}(R)=((3),(3),(2),(2),(2),(2),(1),(1),(1))$ and $\gamma=(3,3,2,2,2,2,1,1,1)$. Similarly, define columns $\left(R^{t}\right)$ to be the sequence of transposes of $\operatorname{rows}(R)$. We have the bijection

$$
\Phi_{\text {rows }(R)}^{-1}: R C\left(\lambda^{t} ; \operatorname{columns}\left(R^{t}\right)\right) \rightarrow L R T(\lambda, \operatorname{rows}(R))=T(\lambda, \gamma)
$$


It is clear from the definitions that there is an inclusion

$$
R C\left(\lambda^{t} ; R^{t}\right) \subseteq R C\left(\lambda^{t} ; \operatorname{columns}\left(R^{t}\right)\right)
$$

Let

$$
b_{R}: R C\left(\lambda^{t} ; R^{t}\right) \rightarrow T(\lambda, \gamma)
$$

be the restriction of the map $\Phi_{\text {rows }(R)}^{-1}$ to the subset $R C\left(\lambda^{t} ; R^{t}\right)$.

Conjecture 10 If $R$ is dominant then $\operatorname{Im} b_{R}=C T(\lambda ; R)$, and $b_{R} \circ \Phi_{R}$ is a bijection $\operatorname{LRT}(\lambda ; R) \rightarrow C T(\lambda ; R)$ sending charge ${ }_{R}$ to charge.

In Section 10 the composite map $L R T(\lambda ; R) \rightarrow C T(\lambda ; R)$ is given without using rigged configurations as an intermediate set.

\section{Symmetry bijections}

Let $R$ and $R^{\prime}$ be any two sequences of rectangles that are rearrangements of each other. It follows immediately from the definitions that $R C(\lambda ; R)=R C\left(\lambda ; R^{\prime}\right)$, so that

$$
R C_{\lambda ; R}(q)=R C_{\lambda ; R^{\prime}}(q)
$$

We now give the symmetry bijections of LR tableaux and a another conjectural description for charge ${ }_{R}$. Fix the sequence of rectangles $R=\left(R_{1}, R_{2}, \ldots, R_{t}\right)$. Let $\mathcal{R}$ be the set of rearrangements of $R . \Sigma_{[t]}$ acts on $\mathcal{R}$ in the obvious way. For $u \in \Sigma_{[t]}$, we wish to define bijections

$$
u_{R}: \operatorname{LRT}(\lambda ; R) \rightarrow \operatorname{LRT}(\lambda ; u R)
$$

that give an action of $\Sigma_{[t]}$ on LR tableaux in the sense that the two bijections $\operatorname{LRT}(\lambda ; R) \rightarrow$ $\operatorname{LRT}(\lambda ; v u R)$ given by $(v \circ u)_{R}$ and $v_{u R} \circ u_{R}$, coincide. When each rectangle in $R$ is a single row, these bijections coincide with the action of the symmetric group on the plactic algebra by the automorphisms of conjugation [25], whose Coxeter generators are sometimes called crystal reflection operators.

Suppose that $u$ is the adjacent transposition $s_{1}=(12)$ and $R=\left(R_{1}, R_{2}\right)$ consists of two rectangles. Let $P \in L R T(\lambda ; R)$. By (2.6), both the sets $L R T(\lambda ; R)$ and $L R T\left(\lambda ;\left(R_{2}, R_{1}\right)\right)$ are singletons, say $\{P\}$ and $\left\{P^{\prime}\right\}$ respectively. In this case the bijection $s_{1}=\left(s_{1}\right)_{R}$ is defined by $s_{1} P=P^{\prime}$, and its inverse (also denoted $s_{1}$ by suppressing the subscript) is given by $s_{1} P^{\prime}=P$. The tableau $P^{\prime}$ can be calculated as follows. Let $A_{1}^{\prime}, A_{2}^{\prime}, K_{1}^{\prime}$ and $K_{2}^{\prime}$ be the subalphabets and canonical tableaux for the sequence of rectangles $s_{1} R=\left(R_{2}, R_{1}\right)$. Clearly $\left.P^{\prime}\right|_{A_{1}^{\prime}}=K_{1}^{\prime}$. The remainder $\left.P^{\prime}\right|_{A_{2}^{\prime}}$ of $P^{\prime}$ is the tableau of shape $\lambda / R_{2}$ obtained by the jeu-de-taquin given by sliding the tableau $K_{2}^{\prime}$ to the southeast into the skew shape $\lambda / R_{1}$ using the order of cells defined by the skew tableau $\left.P\right|_{A_{2}}$.

Next suppose $u=s_{p}$ where $p>1$. Let $B=A_{p} \cup A_{p+1}$. Let $(P, Q)$ be the tableau pair corresponding to the column insertion of the row-reading word of the skew tableau $\left.T\right|_{B}$. 
Since $T \in L R T(\lambda ; R)$, the tableaux $\left.T\right|_{A_{p}}$ and $\left.T\right|_{A_{p+1}}$ are lattice with respect to the alphabets $A_{p}$ and $A_{p+1}$ respectively. Since the lattice condition is invariant under Knuth equivalence, it follows that the restrictions of $P$ to the alphabets $A_{p}$ and $A_{p+1}$ are lattice, so that $P \in \operatorname{LRT}\left(\rho ;\left(R_{p}, R_{p+1}\right)\right)$ in the alphabet $B$, where $\rho$ is the shape of $P$. Let $P^{\prime}$ be the unique tableau in the singleton set $\operatorname{LRT}\left(\rho ;\left(R_{p+1}, R_{p}\right)\right)$ in the alphabet $B$. By [44] [Theorem 1], there is a skew tableau $U$ of the same skew shape as $\left.T\right|_{B}$, whose rowreading word corresponds to the tableau pair $\left(P^{\prime}, Q\right)$ under column insertion. Define $s_{p} T$ be the tableau which agrees with $U$ on the alphabet $B$ and agrees with $T$ on the complement of $B$. Note that $s_{p} T \in L R T\left(\lambda ; s_{p} R\right)$ since latticeness is preserved under Knuth equivalence.

Example 14 Let $p=2$. The subalphabets for the sequence of rectangles $s_{p} R$ are $A_{1}^{\prime}=$ $[1,2], A_{2}^{\prime}=[3,5]$, and $A_{3}^{\prime}=[6,9]$, with $B=[3,9]$. Consider the first tableau $T$ of Example 11. The tableau $s_{2} T$ is computed as follows.

$$
\begin{aligned}
& \begin{array}{lllllllllllllll}
1 & 1 & 1 & 3 & 3 & & & 3 & 3
\end{array} \\
& \begin{array}{lllllll}
2 & 2 & 2 & 4 & &
\end{array}
\end{aligned}
$$

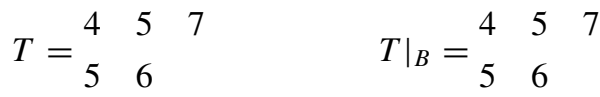

$$
\begin{aligned}
& \begin{array}{lll}
68 & 6 & 8
\end{array} \\
& 929
\end{aligned}
$$

$\operatorname{word}(T)=9.68 .56 .457 .4 .33$

$$
\begin{aligned}
& \begin{array}{lllllllll}
3 & 3 & 7 & 1 & 2 & 6 & 3 & 6 & 6
\end{array} \\
& \begin{array}{llllll}
4 & 4 & 3 & 5 & 4 & 7
\end{array} \\
& P=\begin{array}{ll}
5 & 5 \\
6 & 6
\end{array} \quad Q=\begin{array}{cc}
4 & 8 \\
7 & 10
\end{array} \quad P^{\prime}=\begin{array}{ll}
5 & 8 \\
7 & 9
\end{array} \\
& 89 \\
& 9 \quad 11 \quad 9
\end{aligned}
$$

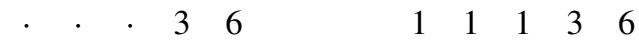

$$
\begin{aligned}
& \text {. } \cdot 4 \quad 2224 \\
& \left.\left(s_{2} T\right)\right|_{B}=\begin{array}{lll}
5 & 6 & 7 \\
7 & 8 &
\end{array} \quad s_{2} T=\begin{array}{lll}
5 & 6 & 7 \\
7 & 8 &
\end{array} \\
& \begin{array}{llll}
8 & 9 & 8 & 9
\end{array} \\
& 9
\end{aligned}
$$

For an arbitrary permutation $u \in \Sigma_{[t]}$, the bijection

$$
u_{R}: \operatorname{LRT}(\lambda ; R) \rightarrow \operatorname{LRT}(\lambda ; u R)
$$


is defined to be the composition $u=s_{a_{1}} s_{a_{2}} \cdots s_{a_{r}}$, where $a_{1} a_{2} \cdots a_{r}$ is a reduced word for $u$. These bijections were chosen with the following property in mind.

Conjecture 11 The following diagram commutes:

$$
\begin{array}{cc}
\operatorname{LRT}(\lambda ; R) \stackrel{u_{R}}{\longrightarrow} & \operatorname{LRT}(\lambda ; u R) \\
\Phi_{R} \downarrow & \downarrow \Phi_{u} R \\
R C\left(\lambda^{t} ; R^{t}\right)= & R C\left(\lambda^{t} ; u\left(R^{t}\right)\right)
\end{array}
$$

In particular, the bijection $u_{R}$ is independent of the reduced word of $u$, and the bijections of the form $u_{R}$ define an action of $\Sigma_{[t]}$ on the collection $\mathcal{R}$ of $L R$ tableaux.

When each rectangle in $R$ is a single row, a version of this result is stated in [3][(2.17)].

It suffices to prove Conjecture 11 for adjacent transpositions $s_{p}$. We show that this conjecture may be reduced to another conjecture on evacuation. Let $e v=e_{[n]}$ denote the evacuation involution on tableaux in the alphabet $[n]$; it is defined by the conditions

$$
\operatorname{shape}\left(\left.\operatorname{ev}(T)\right|_{[k]}\right)=\operatorname{shape}\left(P\left(\left.T\right|_{[n+1-k, n]}\right)\right)
$$

for all $1 \leq k \leq n$. Since latticeness is preserved by Knuth equivalence, ev restricts to a bijection $\operatorname{LRT}(\lambda ; R) \rightarrow \operatorname{LRT}(\lambda ; \operatorname{rev}(R))$ where $\operatorname{rev}(R)$ is the reverse of $R$.

Consider also the involution $\theta$ on $R C(\lambda ; R)=R C(\lambda ; \operatorname{rev}(R))$ given by $(\nu, L) \mapsto(\nu, J)$ where

$$
L_{s}^{k}+J_{s}^{k}=P_{k, v_{s}^{k}}(v)
$$

for all $k \geq 1,1 \leq s \leq \ell\left(v^{k}\right)$. This is slightly sloppy since the labels have to be reordered to satisfy the formal definition of a rigging. The involution $\theta$ complements each coquantum number $L_{s}^{k}$ with respect to its maximum possible value.

Conjecture 12 The following diagram commutes:

$$
\begin{array}{ccc}
L R(\mid \lambda ; R) & \mathrm{ev} & L R(\lambda ; \operatorname{rev}(R)) \\
\Phi_{R} \downarrow & & \downarrow \Phi_{\operatorname{rev}(R)} \\
R C\left(\lambda^{t} ; R^{t}\right) & \longrightarrow & R C\left(\lambda^{t} ; \operatorname{rev}\left(R^{t}\right)\right)
\end{array}
$$

When each rectangle of $R$ is a single row, a similar assertion is made in [18].

\section{Lemma 6 Conjecture 11 follows from Conjecture 12.}

Proof: We may assume that $u=s_{p}$. If $p=1$ this may be verified directly using the definition of the map $\Phi_{R}$. So suppose $p>1$. Again by the definition of $\Phi_{R}$, it suffices to assume 
that $t=p+1$, that is, $s_{p}$ exchanges the last two rectangles in $R$. We have

$$
\mathrm{evs}_{p} T=s_{1} \mathrm{ev} T
$$

for all $T \in \operatorname{LRT}(\lambda ; R)$, which holds since latticeness is preserved by Knuth equivalence. Using the fact that ev is an involution, that Conjecture 11 holds for $s_{1}$, and assuming Conjecture 12, it follows that Conjecture 11 also holds for $s_{p}$.

Next is another conjectural characterization of the statistic charge ${ }_{R}$ on LR tableaux; its definition requires Conjecture 11. The notation in the definition of the bijection $s_{p}$ is used here. Let $T \in L R T(\lambda ; R)$. Define the statistic $d_{p, R}(T)$ to be the number of cells in $P=P\left(\left.T\right|_{A_{p} \cup A_{p+1}}\right)$ that lie to the right of the $c$-th column, where $c=\max \left(\mu_{p}, \mu_{p+1}\right)$. Compare this to (2.7). Let

$$
d_{R}(T)=\sum_{p=1}^{t-1}(t-p) d_{p, R}(T) .
$$

The alternate definition for charge ${ }_{R}$ (valid for any $R$ ) is:

$$
\operatorname{charge}_{R}(T)=\frac{1}{t !} \sum_{u \in \Sigma_{[t]}} d_{u R}(u T)
$$

(see Conjecture 7). It is not clear why this quantity should be an integer. When each rectangle in $R$ is a single row, (7.1) specializes to the formula for the charge given in [21]. By definition

$$
\operatorname{charge}_{u R}(u T)=\operatorname{charge}_{R}(T)
$$

for all $T \in \operatorname{LRT}(\lambda ; R)$ and $u \in \Sigma_{[t]}$.

Example 15 Let us use (7.1) to compute charge ${ }_{R}$ for the tableau $T$ of Example 14. It is necessary to compute the entire orbit of $T$ under the action of $\Sigma_{[3]}$.

$$
T=\begin{array}{lllll}
1 & 1 & 1 & 3 & 3 \\
2 & 2 & 2 & 4 & \\
4 & 5 & 7 & & \\
5 & 6 & & & \\
6 & 8 & & \\
9 & & &
\end{array}
$$




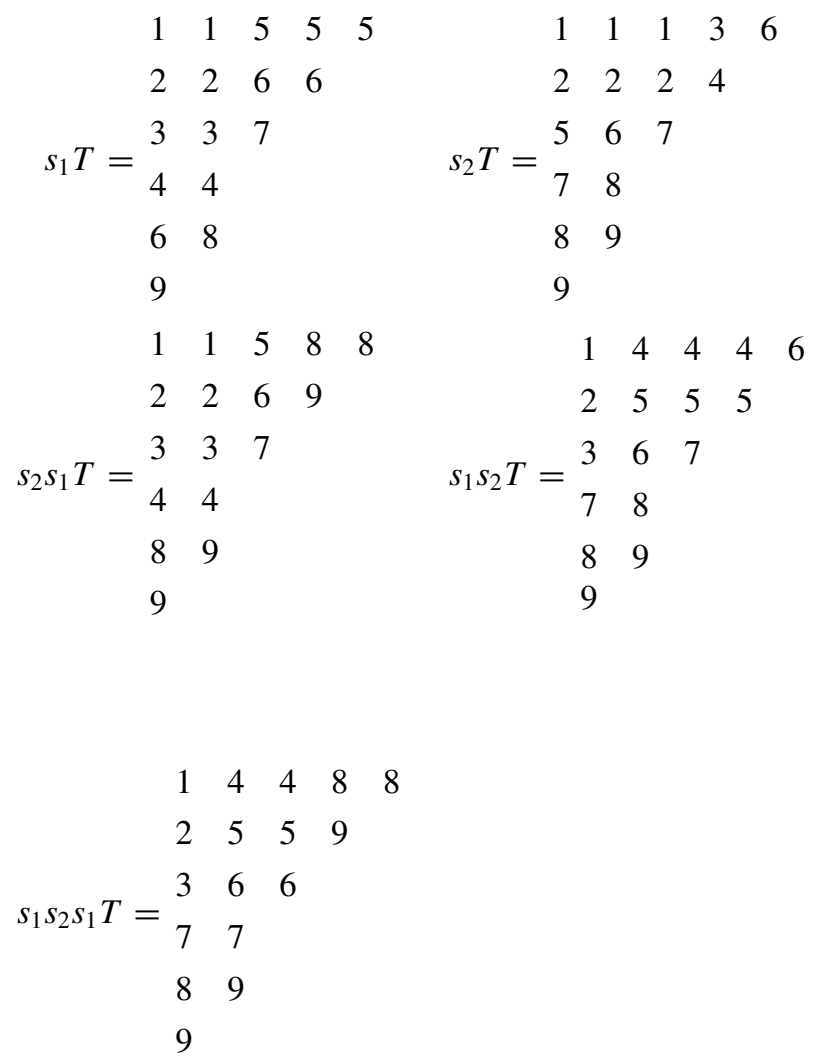

We now give the statistics $d_{1, u R}, d_{2, u R}$, and $d_{u R}$ for each of the tableaux $u T$, with $t=3$.

$\begin{array}{cccc}u & d_{1, u R} & d_{2, u R} & d_{u R} \\ \mathrm{id} & 3 & 1 & 7 \\ s_{1} & 3 & 0 & 6 \\ s_{2} & 2 & 1 & 5 \\ s_{2} s_{1} & 3 & 0 & 6 \\ s_{1} s_{2} & 2 & 1 & 5 \\ s_{1} s_{2} s_{1} & 3 & 1 & 7\end{array}$

So charge $_{R}(T)=(7+6+5+6+5+7) / 3 !=6$.

Finally we give the symmetry bijection for catabolizable tableaux. Let $R$ and $R^{\prime}$ be sequences of rectangles that rearrange each other and $u \in \Sigma_{[n]}$ the shortest permutation such that $\gamma\left(R^{\prime}\right)=u \gamma(R)$. From [3] it follows that the following diagram commutes, where 
$u$ acts by an automorphism of conjugation:

$$
\begin{array}{ccc}
T(\lambda, \gamma(R)) & \stackrel{u}{\longrightarrow} & T\left(\lambda, \gamma\left(R^{\prime}\right)\right) \\
\Phi_{\operatorname{rows}(R) \downarrow} & & \downarrow \Phi_{\operatorname{rows}\left(R^{\prime}\right)} \\
\left.R c\left(\lambda^{t} ; \text { columns } R^{t}\right)\right) & = & R C\left(\lambda^{t} ;\left(\operatorname{columns}\left(R^{\prime}\right)^{t}\right)\right)
\end{array}
$$

Conjecture 10 implies the following result.

Conjecture 13 The automorphism of conjugation $u$ restricts to a bijection

$$
\operatorname{Im} b_{R} \rightarrow \operatorname{Imb} b_{R^{\prime}}
$$

so that the diagram commutes:

$$
\begin{array}{ccc}
R c\left(\lambda^{t} ; R^{t}\right) & = & R C\left(\lambda^{t} ;\left(R^{\prime}\right)^{t}\right) \\
b_{R} \downarrow & & \downarrow b_{R^{\prime}} \\
T(\lambda, \gamma(R)) \underset{u}{\longrightarrow} & T\left(\lambda, \gamma\left(R^{\prime}\right)\right)
\end{array}
$$

In particular, if both $R$ and $R^{\prime}$ are dominant, then $u$ restricts to a bijection

$$
C T(\lambda ; R) \rightarrow C T\left(\lambda ; R^{\prime}\right)
$$

The conjecture is trivial when each rectangle in $R$ is a single row, but is quite interesting even when each rectangle is a single column (in which case the permutation $u$ is the identity).

Example 16 Let $R^{\prime}=s_{1} R=((2,2,2,2),(3,3),(1,1,1))$. Then the permutation $u$ of minimal length sending $\gamma(R)$ to $\gamma\left(R^{\prime}\right)$ is given by $u=561234789$, which has the reduced word 43215432. Let $S$ be the first tableau in Example 13. Here the operators $s_{i}$ are the automorphisms of conjugation, or equivalently the rectangle-switching bijections for the appropriate sequences of one-rowed rectangles.

$$
\begin{aligned}
& \begin{array}{lllll}
1 & 1 & 1 & 5 & 6
\end{array} \\
& \begin{array}{lllll}
1 & 1 & 5 & 5 & 6
\end{array} \\
& 2226 \\
& \begin{array}{llll}
2 & 2 & 6 & 6
\end{array} \\
& S=\begin{array}{lll}
3 & 3 & 7 \\
4 & 4
\end{array} \\
& s_{4} s_{3} s_{2} s_{1} s_{5} s_{4} s_{3} s_{2} S=\begin{array}{ll}
3 & 3 \\
4 & 4
\end{array} \\
& 58 \\
& 58 \\
& 9 \\
& 9
\end{aligned}
$$

\section{Duality bijections}

This section uses the notation of Subsection 2.7. Fix an integer $k$ such that $k \geq \lambda_{1}$ and $k \geq \mu_{i}$ for all $1 \leq i \leq t$. Let $(\tilde{R})^{t}$ denote the sequence of rectangles whose $i$-th partition is $\left(\tilde{R}_{i}\right)^{t}$, a $\left(k-\mu_{i}\right) \times \eta_{i}$ rectangle. 
First we define a duality bijection for rigged configurations.

Proposition 7 There is a bijection of admissible configurations

$$
\begin{aligned}
C\left(\lambda^{t} ; R^{t}\right) & \rightarrow C\left((\tilde{\lambda})^{t} ;(\tilde{R})^{t}\right) \\
v=\left(v^{1}, v^{2}, \ldots, v^{k-1},(), \ldots\right) & \mapsto \tilde{v}=\left(v^{k-1}, v^{k-2}, \ldots, v^{1},(), \ldots\right)
\end{aligned}
$$

Furthermore, for every $1 \leq i \leq k-1$ and $j \geq 1, P_{i, j}(v)=P_{k-i, j}(\tilde{v})$. In particular the above bijection on configurations induces a cocharge-preserving map of rigged configurations $R C\left(\lambda^{t} ; R^{t}\right) \rightarrow R C\left((\tilde{\lambda})^{t} ;(\tilde{R})^{t}\right)$ such that $(v, L) \mapsto(\tilde{v}, \tilde{L})$ where $\tilde{L}_{s}^{p}=\tilde{L}_{s}^{k-p}$ for all $1 \leq p<k$ and $1 \leq s \leq \ell\left(v^{p}\right)=\ell\left(\tilde{v}^{k-p}\right)$.

In other words, the bijection merely replaces the first $k-1$ labelled partitions of $(v, L)$ (the rest are empty) by the reverse sequence of labelled partitions. The proof is straightforward. It follows immediately that

$$
R C_{\lambda^{t} ; R^{t}}(q)=R C_{(\tilde{\lambda})^{t} ;(\tilde{R})^{t}}(q)
$$

Next we consider the duality bijections for LR tableaux. Let $T$ be any tableau of shape $\lambda$ and content $\gamma$. Define the dual tableau $\tilde{T}$ of $T$ (with respect to the $n \times k$ rectangle) to be the unique tableau of shape $\tilde{\lambda}$ such that the $j$ th column of $\tilde{T}$ is the set complement within the interval $[n]$, of the $(k+1-j)$-th column of $T$. Clearly $\tilde{T}$ has content $\tilde{\gamma}=$ $\left(k-\gamma_{n}, k-\gamma_{n-1}, \ldots, k-\gamma_{1}\right)$.

Example 17 In our examples, we have $n=9$ and

$$
\lambda=(5,4,3,2,2,1,0,0,0) \quad R=((3,3),(2,2,2,2),(1,1,1))
$$

Let $k=5$. Then

$$
\begin{aligned}
\tilde{\lambda} & =(5,5,5,4,3,3,2,1,0) \\
\tilde{R} & =((2,2),(3,3,3,3),(4,4,4))
\end{aligned}
$$

The tableau $T \in \operatorname{LRT}(\lambda ; R)$ is sent to $\tilde{T} \in \operatorname{LRT}(\tilde{\lambda} ; \tilde{R})$.

$$
\begin{aligned}
& \begin{array}{lllll}
1 & 1 & 3 & 3 & 3
\end{array} \\
& \begin{array}{llllllllll}
1 & 1 & 1 & 3 & 3 & 2 & 2 & 4 & 4 & 7
\end{array} \\
& \begin{array}{lllllllll}
2 & 2 & 2 & 4 & 4 & 5 & 5 & 7 & 8
\end{array} \\
& T=\begin{array}{lll}
4 & 5 & 7 \\
5 & 6
\end{array} \quad \quad \tilde{T}=\begin{array}{llll}
5 & 6 & 6 & 9 \\
6 & 7 & 8 &
\end{array} \\
& \begin{array}{lllll}
6 & 8 & 7 & 8 & 9
\end{array} \\
& 9 \quad 8 \quad 9
\end{aligned}
$$


The duality map respects Knuth equivalence in the following sense. Let $b$ be a strictly decreasing word in the alphabet $[n]$. Let $\tilde{b}$ be the strictly decreasing word whose letters are complementary in $[n]$ to those of $b$.

Proposition 8 ([31]) Let $a, b, c$, and d be strictly decreasing words in the alphabet $[n]$. Then $a b \sim_{K}$ cd if and only if $\tilde{b} \tilde{a} \sim_{K} \tilde{d} \tilde{c}$ where $\sim_{K}$ denotes Knuth equivalence. In particular, for any skew tableau $T, P(\tilde{T})=\widetilde{P(T)}$, where, for the purpose of applying the duality map, all tableaux are regarded as having the same number of (possibly empty) columns.

The duality bijection on tableaux also restricts to a map from LR tableaux to LR tableaux.

Proposition 9 The bijection $T \mapsto \tilde{T}$ restricts to a bijection

$$
\operatorname{LRT}(\lambda ; R) \rightarrow \operatorname{LRT}(\tilde{\lambda} ; \tilde{R}) .
$$

Furthermore, for every $T \in \operatorname{LRT}(\lambda ; R)$ we have

$$
\operatorname{charge}_{R}(T)=\operatorname{charge}_{\tilde{R}}(\tilde{T}),
$$

using (7.1) as the definition of charge . .

Proof: Let $T$ be a tableau of shape $\lambda$ and content $\gamma$. The following are equivalent:

1. $T \in \operatorname{LRT}(\lambda ; R)$.

2. $\left.T\right|_{A_{i}}$ is lattice in the alphabet $A_{i}$ for all $i$.

3. For all $0 \leq j \leq k$ and all $i$, the last $j$ columns of $\left.T\right|_{A_{i}}$ have partition content in the alphabet $A_{i}$.

4. For all $0 \leq j \leq k$ and all $i$, the first $j$ columns of $\tilde{T}_{A_{i}}$ has antipartition content in the alphabet $A_{i}$.

5. For all $0 \leq j \leq k$ and all $i$, the last $k-j$ columns of $\left.\tilde{T}\right|_{A_{i}}$ have partition content in the alphabet $A_{i}$.

6. $\left.\tilde{T}\right|_{A_{i}}$ is lattice in the alphabet $A_{i}$ for all $i$.

7. $\tilde{T} \in \operatorname{LRT}(\tilde{\lambda} ; \tilde{R})$.

The equivalence of the first three and last three assertions follow by definition. The equivalence of items 3 and 4 follows from the definition of $\tilde{T}$. The equivalence of 4 and 5 follows from the fact that for each letter $x \in A_{i}$, the letter $x$ appears with total multiplicity $\mu_{i}$.

To verify (8.1), it suffices to show that for all $1 \leq p \leq t-1$ and all $u$,

$$
d_{p, u R}(u T)=d_{p, u \tilde{R}}(u \tilde{T})
$$

It is immediate that $u \tilde{R}=\widetilde{u R}$. It suffices to establish the identities

$$
\begin{aligned}
d_{p, R}(T) & =d_{p, \tilde{R}}(\tilde{T}) \quad \text { for all } R \\
u \tilde{T} & =\widetilde{u T} \quad \text { for all } u .
\end{aligned}
$$


To prove (8.2), one can reduce to the case $p=1$ and $t=2$ by Proposition 8 . Without loss of generality assume that $\mu_{1} \geq \mu_{2}$. Let $\alpha$ and $\beta$ be the left and right partitions given by slicing the skew shape $\lambda / R_{1}$ vertically just after the $\mu_{1}$-th column. The Littlewood-Richardson rule implies that the 180 degree rotation of $\alpha$ fits together with $\beta$ to form the rectangle $R_{2}$. We have

$$
\begin{aligned}
d_{1, R}(T) & =\mu_{2} n-\left(\lambda_{1}^{t}+\cdots+\lambda_{\mu_{2}}^{t}\right) \\
& =\tilde{\lambda}_{k}^{t}+\tilde{\lambda}_{k-1}^{t}+\cdots+\tilde{\lambda}_{k+1-\mu_{2}}^{t} \\
& =d_{1, \tilde{R}}(\tilde{T})
\end{aligned}
$$

proving (8.2)

It suffices to prove (8.3) when $u$ is an adjacent transposition $s_{p}$. Let $B=A_{p} \cup A_{p+1}$. By the definitions it follows immediately that $s_{p} \tilde{T}$ and $\widetilde{s_{p} T}$ agree when restricted to the complement of the alphabet $B$. It remains to show that the restrictions of the two tableaux to $B$ agree. By Proposition 8 , we may assume that $p=1$ and $t=2$. But this case follows immediately since taking the dual tableau and applying $s_{1}$ both send LR tableaux to LR tableaux, and all of the relevant sets of LR tableaux are singletons.

It follows from (7.1) that

$$
\operatorname{LRT}_{\lambda ; R}(q)=L R T_{\tilde{\lambda} ; \tilde{R}}(q)
$$

Moreover,

Conjecture 14 The following diagram commutes

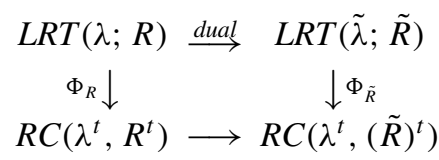

where the bottom map is given in Prop. 7.

To discuss the duality bijection for catabolizable tableaux, we give a modified definition that slices the tableaux vertically rather than horizontally. In this section let us refer to $R$-catabolizability as $R$-row catabolizability and to the $R_{1}$-catabolism as the $R_{1}$-row catabolism.

Let $S$ be a tableau of partition shape with $\left.S\right|_{A_{1}}=K_{1}$. Let $S_{l}$ and $S_{r}$ be the left and right subtableaux obtained by slicing the skew tableau $S-K_{1}$ vertically just after the $\mu_{1}$-th column. Define the $R_{1}$-column catabolism CCat $R_{1}(S)$ of $S$ by $P\left(S_{r} S_{l}\right)$. Say that $S$ is $R$-column catabolizable if $\left.S\right|_{A_{1}}=K_{1}$ and $\operatorname{CCat}_{R_{1}}(S)$ is $\hat{R}$-column catabolizable, where $\hat{R}=\left(R_{2}, R_{3}, \ldots\right)$. Let $C C T(\lambda ; R)$ denote the set of $R$-column catabolizable tableaux of shape $\lambda$.

Proposition $10 S$ is R-column catabolizable if and only if $\tilde{S}$ is $\tilde{R}$-column catabolizable. 
Proof: Fix a sufficiently large number $k$. All dual tableaux will be taken with respect to $k$ columns. Note first that $\left.S\right|_{A_{1}}=K_{1}$ if and only if $\left.\tilde{S}\right|_{A_{1}}$ is the tableau of shape $\tilde{R}_{1}$ whose $i$-th row consists of $k-\mu_{1}$ copies of the letter $i$ for all $1 \leq i \leq \eta_{1}$. Then Proposition 8 implies that the dual of the tableau $\mathrm{CCat}_{R_{1}}(S)$ with respect to the alphabet $[n]-A_{1}$, is equal to the tableau $\mathrm{CCat}_{\tilde{R}_{1}}(\tilde{S})$. The result follows by induction.

The following conjecture connects the two kinds of catabolizability using the images of the maps $b_{R}$.

Conjecture 15 Suppose $R$ is a sequence of rectangles such that $R^{t}$ is dominant. Then $\operatorname{Im} b_{R}=C C T(\lambda ; R)$.

Using Conjectures 13 and 15 one obtains a duality bijection for row catabolizable tableaux using automorphisms of conjugation and the tableau duality bijection.

Example 18 If the hypothesis of Conjecture 15 is not satisfied then $C C T(\lambda ; R)$ could be too large. For example, let $\lambda=(2,2), R=((1,1,1),(1))$, and $R^{\prime}=((1),(1,1,1))$. Then $\operatorname{CCT}(\lambda ; R)$ is empty. But $C C T\left(\lambda ; R^{\prime}\right)$ is not; it is equal to $\operatorname{CCT}((2,2) ;((1,1)$, $(1,1)))$.

\section{Transpose bijections}

A bijection $\operatorname{LRT}(\lambda ; R) \rightarrow \operatorname{LRT}\left(\lambda^{t} ; R^{t}\right)$ is given by the relabelling which sends $T$ to the transpose of the tableau obtained from $T$ by replacing the $j$-th occurrence (from the left) of the letter $\eta_{1}+\eta_{2}+\cdots+\eta_{i-1}+k$ by the letter $\mu_{1}+\mu_{2}+\cdots+\mu_{i-1}+j$, for all $i$, $1 \leq j \leq \mu_{i}$ and $1 \leq k \leq \eta_{i}$. We call this map the LR transpose.

Example $19 \lambda^{t}=(6,5,3,2,1)$ and $R_{1}^{t}=(2,2,2), R_{2}^{t}=(4,4)$ and $R_{3}^{t}=(3)$. The set $L R T\left(\lambda^{t} ; R^{t}\right)$ is given by the following four tableaux, which are the images under the $L R$ transpose map of the four tableaux of $L R T(\lambda ; R)$ listed in Example 11.

$\begin{array}{llllllllllllllllllllllll}1 & 1 & 4 & 4 & 4 & 6 & 1 & 1 & 4 & 4 & 4 & 6 & 1 & 1 & 4 & 4 & 4 & 4 & 1 & 1 & 4 & 4 & 4 & 4 \\ 2 & 2 & 5 & 5 & 6 & & 2 & 2 & 5 & 5 & 5 & & 2 & 2 & 5 & 5 & 6 & & 2 & 2 & 5 & 5 & 5 & \\ 3 & 3 & 6 & & & & 3 & 3 & 6 & & & & 3 & 3 & 6 & & & & 3 & 3 & 6 & & & \\ 4 & 5 & & & & & 5 & 5 & & & & & 5 & 5 & & & & & 5 & 6 & & & \\ 5 & & & & & & 6 & & & & & & 6 & & & & & & 6 & & & & \end{array}$

For rigged configurations, we wish to define a bijection $R C(\lambda ; R) \rightarrow R C\left(\lambda^{t} ; R^{t}\right)$ (called the RC-transpose) sending $(v, L) \rightarrow(\hat{v}, \hat{L})$ with the property that

$$
\operatorname{cocharge}(\nu, L)=n(R)-\operatorname{cocharge}(\hat{v}, \hat{L}) .
$$

The bijection on configurations is most easily defined using a variant of the original construction. Let $v$ be an admissible $(\lambda ; R)$ configuration. Recall that $v^{0}$ is the empty 
partition and $\alpha_{k, n}$ is the size of the $n$-th column of the partition $v^{k}$. Define the matrix $\left(m_{i, j}\right)$ by

$$
m_{i, j}=\alpha_{i-1, j}-\alpha_{i, j}
$$

for $i, j \geq 1$, or equivalently,

$$
\alpha_{i, j}=-\sum_{k=1}^{i} m_{k, j}
$$

The matrix $\left(m_{i, j}\right)$ will be used in place of the configuration $v$. Let us calculate the row and column sums of the matrix $\left(m_{i, j}\right)$ and the cocharge of $v$ in terms of the $m_{i, j}$. Let $\theta$ be the indicator function for nonnegative numbers:

$$
\theta(x)= \begin{cases}1 & \text { if } \quad x \geq 0 \\ 0 & \text { if } \quad x<0\end{cases}
$$

Since $\alpha_{0, j}=0$ for all $j$ and $\alpha_{i, j}=0$ for large $i$,

$$
\sum_{i} m_{i, j}=\sum_{i}\left(\alpha_{i-1, j}-\alpha_{i, j}\right)=0 .
$$

Using (3.1) and the notation $r_{+}=\max (r, 0)$, we have

$$
\begin{aligned}
\sum_{j} m_{i, j} & =\sum_{j}\left(\alpha_{i-1, j}-\alpha_{i, j}\right)=\left|v^{i-1}\right|-\left|v^{i}\right| \\
& =\sum_{j>i-1}\left(\lambda_{j}-\sum_{a} \mu_{a}\left(\eta_{a}-(i-1)\right)_{+}\right)-\sum_{j>i}\left(\lambda_{j}-\sum_{a} \mu_{a}\left(\eta_{a}-i\right)_{+}\right) \\
& =\lambda_{i}-\sum_{a} \mu_{a} \theta\left(\eta_{a}-i\right)
\end{aligned}
$$

The cocharge (3.3) can be rewritten as

$$
\begin{aligned}
\operatorname{cocharge}(v) & =\sum_{k, n \geq 1} \alpha_{k, n}\left(\alpha_{k, n}-\alpha_{k+1, n}\right) \\
& =\sum_{k, n \geq 1}\left(-m_{1, n}-m_{2, n}-\cdots-m_{k, n}\right) m_{k+1, n} \\
& =-\sum_{\substack{n \geq 1 \\
j>k \geq 1}} m_{k, n} m_{j, n} \\
& =-1 / 2 \sum_{\substack{n \geq 1 \\
j \neq k \geq 1}} m_{k, n} m_{j, n}
\end{aligned}
$$




$$
\begin{aligned}
& =1 / 2 \sum_{k, n \geq 1} m_{k, n}^{2}-1 / 2 \sum_{j, k, n \geq 1} m_{k, n} m_{j, n} \\
& =1 / 2 \sum_{k, n \geq 1} m_{k, n}^{2} \\
& =\sum_{k, n \geq 1}\left(\begin{array}{c}
m_{k, n} \\
2
\end{array}\right),
\end{aligned}
$$

where the last two equalities hold by (9.4). Define the matrix $\hat{m}_{i, j}$ by

$$
\hat{m}_{i, j}=-m_{j, i}+\theta\left(\lambda_{j}-i\right)-\sum_{a} \theta\left(\mu_{a}-i\right) \theta\left(\eta_{a}-j\right)
$$

Observe that this process is an involution. Applying this process to $\left(\hat{m}_{i, j}\right)$ with respect to the pair $\left(\lambda^{t} ; R^{t}\right)$,

$$
\begin{aligned}
\hat{\hat{m}}_{i, j}= & -\hat{m}_{j, i}+\theta\left(\lambda_{j}^{t}-i\right)-\sum_{a} \theta\left(\eta_{a}-i\right) \theta\left(\mu_{a}-j\right) \\
= & -\left(-m_{i, j}+\theta\left(\lambda_{i}-j\right)-\sum_{a} \theta\left(\mu_{a}-j\right) \theta\left(\eta_{-} i\right)\right)+\theta\left(\lambda_{j}^{t}-i\right) \\
& -\sum_{a} \theta\left(\eta_{a}-i\right) \theta\left(\mu_{a}-j\right) \\
= & m_{i, j}+\theta\left(\lambda_{j}^{t}-i\right)-\theta\left(\lambda_{i}-j\right)=m_{i, j}
\end{aligned}
$$

Note that in (9.7) the sum over $a$ is equal to $r_{j, i}(R)$, the number of rectangles in $R$ that contain the cell $(j, i)$. We must show that the matrix $\left(\hat{m}_{i, j}\right)$ corresponds to an admissible configuration of type $\left(\lambda^{t} ; R^{t}\right)$. Let $\hat{\alpha}_{i, j}, \hat{v}_{j}^{i}, P_{i, j}(\hat{v})$, and $m_{i}\left(\hat{v}^{j}\right)$ denote the analogous quantities involving $\hat{m}_{i, j}$ in place of $m_{i, j}$. The first step is to show that each $\hat{v}^{i}$ is a partition, that is, $m_{j}\left(\hat{v}^{i}\right) \geq 0$ for all $i, j \geq 1$. We have

$$
\begin{aligned}
m_{j}\left(\hat{v}^{i}\right)= & \hat{\alpha}_{i, j}-\hat{\alpha}_{i, j+1} \\
= & \sum_{k=1}^{i}\left(-\hat{m}_{k, j}+\hat{m}_{k, j+1}\right) \\
= & \sum_{k=1}^{i}\left(m_{j, k}-m_{j+1, k}-\theta\left(\lambda_{j}-k\right)+\theta\left(\lambda_{j+1}-k\right)\right. \\
& +\sum_{a} \theta\left(\mu_{a}-k\right)\left(\theta\left(\eta_{a}-j\right)-\theta\left(\eta_{a}-(j+1)\right)\right) \\
= & \sum_{k=1}^{i}\left(\alpha_{j-1, k}-\alpha_{j, k}-\alpha_{j, k}+\alpha_{j+1, k}\right)-\min \left(\lambda_{j}, i\right) \\
& +\min \left(\lambda_{j+1}, i\right)+\sum_{a} \min \left(\mu_{a}, i\right) \delta_{\eta_{a}, j}
\end{aligned}
$$




$$
\begin{aligned}
= & Q_{i}\left(v^{j-1}\right)-2 Q_{i}\left(v^{j}\right)+Q_{i}\left(v^{j+1}\right)+\sum_{a} \min \left(\mu_{a}, i\right) \delta_{\eta_{a}, j} \\
& -\min \left(\lambda_{j}, i\right)+\min \left(\lambda_{j+1}, i\right) \\
= & P_{j, i}(v)-\min \left(\lambda_{j}, i\right)+\min \left(\lambda_{j+1}, i\right)
\end{aligned}
$$

We require the following technical result on vacancy numbers, whose proof is in the appendix. Recall that $m_{n}(\rho)$ is the number of parts of the partition $\rho$ of size $n$.

Lemma 11 Let $v$ be a configurationof type $(\lambda ; R)$. The following are equivalent.

1. $v$ is admissible, that is, $P_{k, n}(v) \geq 0$ for all $k, n \geq 1$.

2. For every $k, n \geq 1$, if $m_{n}\left(v^{k}\right)>0$ then $P_{k, n}(v) \geq 0$.

3. For every $k, n \geq 1$,

$$
P_{k, n}(\nu) \geq \min \left(\lambda_{k}, n\right)-\min \left(\lambda_{k+1}, n\right)
$$

Moreover, if $v$ is admissible then

$$
m_{n}\left(v^{k}\right)=0 \quad \text { whenever } n>\lambda_{k+1} .
$$

From (9.8), Lemma 11, and the admissibility of $v$, it follows that $\hat{v}^{i}$ is a partition for all $i \geq 1$. Next it is verified that $\hat{v}$ is a configuration of type $\left(\lambda^{t} ; R^{t}\right)$.

$$
\begin{aligned}
\left|\hat{v}^{i}\right| & =\sum_{j} \hat{\alpha}_{i, j}=\sum_{j} \sum_{k=1}^{i}-\hat{m}_{k, j} \\
& =\sum_{j} \sum_{k=1}^{i}\left(m_{j, k}-\theta\left(\lambda_{j}-k\right)+\sum_{a} \theta\left(\mu_{a}-k\right) \theta\left(\eta_{a}-j\right)\right) \\
& =\sum_{k=1}^{i} \sum_{j} m_{j, k}-\sum_{j} \min \left(\lambda_{j}, i\right)+\sum_{a} \min \left(\mu_{a}, i\right) \eta_{a} \\
& =-\sum_{j=1}^{i} \lambda_{j}^{t}+\sum_{a} \min \left(\mu_{a}, i\right) \eta_{a}
\end{aligned}
$$

by (9.7) and (9.4). Comparing this with (3.1), $\hat{v}$ is a configuration of type $\left(\lambda^{t} ; R^{t}\right)$, since $R^{t}$ is obtained from $R$ by switching the roles of $\mu$ and $\eta$. Finally it must be verified that $\hat{v}$ is admissible. Since the map $m \mapsto \hat{m}$ is involutive as a map of matrices, it is valid to apply the formula (9.8) to $\hat{v}$ instead of $v$, obtaining

$$
P_{i, j}(\hat{v})=m_{i}\left(v^{j}\right)+\min \left(\lambda_{i}^{t}, j\right)-\min \left(\lambda_{i+1}^{t}, j\right) \geq m_{i}\left(v^{j}\right) \geq 0 .
$$

Therefore the map $\left(m_{i, j}\right) \mapsto\left(\hat{m}_{i, j}\right)$ defines a bijection $C(\lambda ; R) \rightarrow C\left(\lambda^{t} ; R^{t}\right)$. 
This map is extended to riggings as follows. By Lemma 11 and (9.8), the map $m \mapsto \hat{m}$ has the additional property that

$$
\text { if } m_{n}\left(v^{k}\right)>0 \quad \text { then } \quad P_{k, n}(v)=m_{k}\left(\hat{v}^{n}\right) .
$$

Applying (9.11) to the inverse map, it follows that

$$
\text { if } m_{k}\left(\hat{v}^{n}\right)>0 \quad \text { then } \quad P_{n, k}(\hat{v})=m_{n}\left(\nu^{k}\right) .
$$

To show that the two sets of rigged configurations $R C(\lambda ; R)$ and $R C\left(\lambda^{t} ; R^{t}\right)$ have the same cardinality, it suffices to show that the rectangle of height $m_{n}\left(v^{k}\right)$ and width $P_{k, n}(v)$ and the rectangle of height $m_{k}\left(\hat{v}^{n}\right)$ and width $P_{n, k}(\hat{v})$, are either transposes of each other or are both empty. But this follows from (9.11) and (9.12).

Let us give a specific bijection between the riggings. Let $(\nu, L) \in R C(\lambda ; R)$. Let $\hat{v}$ be the admissible configuration of type $\left(\lambda^{t} ; R^{t}\right)$ given by (9.7). Note that a rigging $L$ of $v$ determines, for each pair $k, n \geq 1$, a partition $\rho_{k, n}(\nu, L)$ inside a rectangle of height $m_{n}\left(v^{k}\right)$ and width $P_{k, n}(v)$ given by the the labels of the parts of $v^{k}$ of size $n$.

Let $\hat{L}$ be the rigging of the configuration $\hat{v}$ such that $\rho_{n, k}(\hat{v}, \hat{L})$ is the transpose of the complementary partition to $\rho_{k, n}(v, L)$ in the rectangle of height $m_{n}\left(v^{k}\right)$ and width $P_{k, n}(v)$, for all $k, n \geq 1$.

Then the map $(\nu, L) \mapsto(\hat{v}, \hat{L})$ defines the RC-transpose bijection $R C(\lambda ; R) \rightarrow$ $R C\left(\lambda^{t} ; R^{t}\right)$.

\section{Proposition 12}

$$
R C_{\lambda^{t} ; R^{t}}(q)=q^{n(R)} R C_{\lambda ; R}\left(q^{-1}\right)
$$

Proof: It is enough to check that the RC-transpose bijection $(\nu, L) \mapsto(\hat{v}, \hat{L})$ satisfies

$$
\operatorname{cocharge}(v, L)+\operatorname{cocharge}(\hat{v}, \hat{L})=n(R)
$$

(see (2.8)). By the definition of the rigging $\hat{L}$, it is enough to check that

$$
\operatorname{cocharge}(v)+\operatorname{cocharge}(\hat{v})+\sum_{k, n \geq 1} P_{k, n}(v) m_{n}\left(v^{k}\right)=n(R) .
$$

The sum $\sum_{k, n} P_{k, n}(v) m_{n}\left(v^{k}\right)$ is calculated first. For the vacancy numbers,

$$
\begin{aligned}
Q_{n}\left(v^{k-1}\right)-2 Q_{n}\left(v^{k}\right)+Q_{n}\left(v^{k+1}\right) & =\sum_{j=1}^{n}\left(\alpha_{k-1, j}-2 \alpha_{k, j}+\alpha_{k+1, j}\right) \\
& =\sum_{j=1}^{n}\left(m_{k, j}-m_{k+1, j}\right)
\end{aligned}
$$


and

$$
P_{k, n}(v)=\sum_{j=1}^{n}\left(m_{k, j}-m_{k+1, j}\right)+\sum_{a \geq 1} \min \left(\mu_{a}, n\right) \delta_{\eta_{a}, k} .
$$

The multiplicities $m_{n}\left(v^{k}\right)$ are given by

$$
m_{n}\left(v^{k}\right)=\alpha_{k, n}-\alpha_{k, n+1}=\sum_{i=1}^{k}\left(-m_{i, n}+m_{i, n+1}\right) .
$$

The desired sum is given by

$$
\begin{aligned}
\sum_{k, n \geq 1} P_{k, n}(v) m_{n}\left(v^{k}\right)= & \sum_{k, n \geq 1}\left(\sum_{j=1}^{n}\left(m_{k, j}-m_{k+1, j}\right)+\sum_{a} \min \left(\mu_{a}, n\right) \delta_{\eta_{a}, k}\right) \\
& \times \sum_{i=1}^{k}\left(-m_{i, n}+m_{i, n+1}\right) \\
= & \sum_{\substack{k \geq i \geq 1 \\
n \geq j \geq 1}}\left(m_{k, j}-m_{k+1, j}\right)\left(-m_{i, n}+m_{i, n+1}\right) \\
& \left.+\sum_{k \geq i \geq 1} \sum_{n \geq 1} \min \left(\mu_{a}, n\right) \delta_{\eta_{a}, k}\right)\left(-m_{i, n}+m_{i, n+1}\right) \\
= & -\sum_{i, j \geq 1} m_{i, j}^{2}+\sum_{a} \sum_{i=1}^{\eta_{a}} \sum_{n \geq 1} \min _{n}\left(\mu_{a}, n\right)\left(-m_{i, n}-m_{i, n+1}\right) \\
= & -2 \operatorname{cocharge}(v)-\sum_{a} \sum_{i=1}^{\eta_{a}} \sum_{n=1}^{\mu_{a}} m_{i, n} \\
= & -2 \operatorname{cocharge}(v)-\sum_{a} \sum_{i, j \geq 1} m_{i, j} \theta\left(\mu_{a}-j\right) \theta\left(\eta_{a}-i\right) \\
= & -2 \operatorname{cocharge}(v)-\sum_{i, j \geq 1} m_{i, j} r_{i, j}
\end{aligned}
$$

where $r_{i, j}=r_{i, j}(R)$ is as in the definition of $n(R)$. (2.8)

Next the cocharge of the configuration $\hat{v}$ is calculated. By (3.3), (9.6), and (9.7), we have

$$
\operatorname{cocharge}(\hat{v})=\sum_{i, j \geq 1}\left(\begin{array}{c}
\hat{m}_{i, j} \\
2
\end{array}\right)=\left(\begin{array}{c}
-m_{j, i}+\theta\left(\lambda_{j}-i\right)-r_{j, i} \\
2
\end{array}\right) \text {. }
$$

Using the identities $\left(\begin{array}{c}a+b \\ 2\end{array}\right)=\left(\begin{array}{c}a \\ 2\end{array}\right)+\left(\begin{array}{c}b \\ 2\end{array}\right)+a b$ and $\left(\begin{array}{c}-a \\ 2\end{array}\right)=\left(\begin{array}{c}a \\ 2\end{array}\right)+a$, we have

$$
\operatorname{cocharge}(\hat{v})=\sum_{i, j \geq 1}\left(\left(\begin{array}{c}
m_{j, i} \\
2
\end{array}\right)+\left(\begin{array}{c}
\theta\left(\lambda_{j}-i\right) \\
2
\end{array}\right)+\left(\begin{array}{c}
r_{j, i} \\
2
\end{array}\right)\right.
$$




$$
\begin{aligned}
& \left.-m_{j, i} \theta\left(\lambda_{j}-i\right)+m_{j, i} r_{j, i}-\theta\left(\lambda_{j}-i\right) r_{j, i}+m_{j, i}+r_{j, i}\right) \\
= & \operatorname{cocharge}(\nu)+0+n(R)+\sum_{i, j \geq 1}\left(m_{j, i} r_{j, i}\right. \\
& \left.+\left(1-\theta\left(\lambda_{j}-i\right)\right)\left(m_{j, i}+r_{j, i}\right)\right) .
\end{aligned}
$$

Suppose $i$ and $j$ are such that $\theta\left(\lambda_{j}-i\right)=0$, that is, $i>\lambda_{j}$. Then the cell $(j, i)$ is not in the partition shape $\lambda$. Since the number of rigged configurations $|R C(\lambda ; R)|$ is equal to the LR coefficient $L R_{R}^{\lambda}$, each rectangle $R_{a}$ must be contained in $\lambda$. It follows that $r_{j, i}=0$. Eq. (9.10) guarantees that $\alpha_{j-1, i}=0$. Since $i>\lambda_{j} \geq \lambda_{j+1}, \alpha_{j, i}=0$ also holds. By (9.2) $m_{j, i}=0$. It follows that

$$
\operatorname{cocharge}(\hat{v})=\operatorname{cocharge}(v)+n(R)+\sum_{i, j \geq 1} m_{j, i} r_{j, i}
$$

Together with (9.18), this implies (9.15).

The transpose bijections were chosen with the following property in mind.

Conjecture 16 The following diagram commutes:

$$
\begin{array}{cc}
\operatorname{LRT}(\lambda ; R) \stackrel{\text { LR-transpose }}{\longrightarrow} & \operatorname{LRT}\left(\lambda^{t} ; R^{t}\right) \\
\Phi_{R} \downarrow & \downarrow \Phi_{R^{t}} \\
R C\left(\lambda^{t} ; R^{t}\right) \stackrel{\text { RC-transpose }}{\longrightarrow} & R C(\lambda, R)
\end{array}
$$

We now give a map from the $R$-catabolizable tableaux of shape $\lambda$, to the $R$-column catabolizable tableaux of shape $\tilde{\lambda}$, in the case that $R$ is dominant.

Let $S \in C T(\lambda ; R)$. Let $S_{1}=\mathrm{Cat}_{R_{1}}(S)$ and let $Q_{1}=Q_{c}\left(\operatorname{word}\left(S_{+}\right)\right.$word $\left.\left(S_{-}\right)\right)$where word $(T)$ is the row-reading word of the (skew) tableau $T$, and $Q_{c}(w)$ is the Schensted $Q$ symbol for the column insertion of the word $w$ [34]. The image $U \in C T\left(\lambda^{t} ; R^{t}\right)$ of $S$ under our proposed bijection, is uniquely defined by the property that $\operatorname{Cat}(U)=U_{1}$ where $U_{1}$ is the image of $S_{1}$ (which is defined by induction), and that $Q_{1}^{t}=Q\left(\operatorname{word}\left(U_{r}\right) \operatorname{word}\left(U_{l}\right)\right)$, where $Q(a)$ is the Schensted $Q$ symbol for the row insertion of $a$ (see the definition of $\operatorname{CCat}_{R_{1}}(U)$ ).

In practice one first computes the sequence of tableaux $S_{0}=S$ and $S_{i}=\operatorname{Cat}_{R_{i}}\left(S_{i-1}\right)$, together with the recording tableaux $Q_{i}$ coming from the column insertion of the appropriate row-reading words. Then one computes the sequence of tableaux, $\ldots, U_{2}, U_{1}$ by setting $U_{i}$ to be the tableau such that $\operatorname{CCat}_{R_{i}^{t}}\left(U_{i}\right)=U_{i+1}$ with recording tableau $Q_{i}^{t}$. 
Example 20 Let $S$ be the first tableau listed in Example 13. We give the successive catabolisms of $S$ together with the recording tableaux $Q_{i}$.

$$
\begin{aligned}
& \begin{array}{lllll}
1 & 1 & 1 & 5 & 6
\end{array} \\
& 2226 \\
& S=S_{0}=\begin{array}{lll}
3 & 3 & 7 \\
4 & 4
\end{array} \\
& 58 \\
& 9 \\
& \begin{array}{llllll}
3 & 3 & 7 & 1 & 2 & 3
\end{array} \\
& S_{1}=\begin{array}{rrrrr}
4 & 4 & 8 \\
5 & 5 & 9 \\
6 & 6
\end{array} \quad Q_{1}=\begin{array}{rrr}
4 & 5 & 10 \\
6 & 7 & 11 \\
8 & 9 & \\
11 & &
\end{array} \\
& 7 \quad 1 \\
& S_{2}=8 \quad Q_{2}=2 \\
& 9 \quad 3 \\
& S_{3}=\varnothing \quad Q_{3}=\varnothing
\end{aligned}
$$

The sequence of tableaux $U_{i}$ are calculated by "reverse column catabolisms" whose row insertions are recorded by the transposes of the $Q$ s.

$$
\begin{aligned}
& \begin{array}{llll}
U_{3} & =\emptyset & Q_{3}^{t}=\emptyset
\end{array} \\
& U_{2}=\begin{array}{llllll}
6 & 6 & 6 & Q_{2}^{t}=1 & 2 & 3
\end{array} \\
& \begin{array}{llllllll}
4 & 4 & 4 & 4 & 1 & 4 & 6 & 8
\end{array} \\
& U_{1}=\begin{array}{lllllllll}
5 & 5 & 5 & 5 & Q_{1}^{t}=2 & 5 & 7 & 9
\end{array} \\
& \begin{array}{llllll}
6 & 6 & 6 & 3 & 10 & 11
\end{array} \\
& \begin{array}{llllll}
1 & 1 & 4 & 4 & 5 & 6
\end{array} \\
& \begin{array}{lllll}
2 & 2 & 5 & 5 & 6
\end{array} \\
& U=U_{0}=3 \quad 3 \quad 6 \\
& 44 \\
& 5
\end{aligned}
$$

Conjecture 17 The above map gives a bijection $C T(\lambda ; R)$ to $C C T\left(\lambda^{t} ; R^{t}\right)$ when $R$ and $R^{t}$ are dominant.

The essential point to check is that $U$ is in fact semistandard, since it is conceivable that there could be violations of semistandardness between pairs of entries of the form $U\left(i, \mu_{1}\right)$ and $U\left(i, \mu_{1}+1\right)$ for $i>\eta_{1}$. 


\section{Monotonicity maps}

For this section assume that $R \unrhd R^{\prime}$.

From the definitions it follows directly that $R C\left(\lambda^{t} ; R^{t}\right) \subseteq R C\left(\lambda^{t} ;\left(R^{\prime}\right)^{t}\right)$ which is obviously a cocharge-preserving embedding. At the end of this section we give a direct description of a related charge-preserving embedding $\zeta: R C(\lambda ; R) \hookrightarrow R C(\lambda ; \operatorname{rows}(R))$.

A consequence of Conjecture 10 is that if both $R$ and $R^{\prime}$ are dominant, then $C T(\lambda ; R) \subseteq$ $C T\left(\lambda ; R^{\prime}\right)$.

For LR tableaux, we describe embeddings

$$
\theta_{R}^{R^{\prime}}: \operatorname{LRT}(\lambda ; R) \rightarrow \operatorname{LRT}\left(\lambda ; R^{\prime}\right)
$$

where $R \unrhd R^{\prime}$. To define such embeddings it is enough to assume that $R$ covers $R^{\prime}$, that is, for some $k, \tau^{j}(R)=\tau^{j}\left(R^{\prime}\right)$ for all $k \neq k$ and $\tau^{k}(R)$ covers $\tau^{k}\left(R^{\prime}\right)$ in the dominance order. Using the rectangle switching bijections we may assume that $R$ and $R^{\prime}$ have the form

$$
\begin{aligned}
R & =\left(\left(k^{a}\right),\left(k^{b}\right), R_{3}, R_{4}, \ldots\right) \\
R^{\prime} & =\left(\left(k^{a-1},\left(k^{b+1}\right), R_{3}, R_{4}, \ldots\right)\right.
\end{aligned}
$$

where $a>b+1$. The injection is defined as follows; it generalizes the rectangle-switching bijection in Section 7. Let $T \in \operatorname{LRT}(\lambda ; R)$ and $B=[a+b]$. Then $\left.T\right|_{B}$ comprises the set $\operatorname{LRT}\left(\rho ;\left(\left(k^{a}\right),\left(k^{b}\right)\right)\right)$, where $\rho$ is the shape of $\left.T\right|_{B}$. It follows that $\operatorname{LRT}\left(\rho ;\left(\left(k^{a-1}, k^{b+1}\right)\right)\right)$ consists of a single tableau $T^{\prime \prime}$. Let $T^{\prime}$ be defined by $\left.T^{\prime}\right|_{B}=T^{\prime \prime}$ and $\left.T^{\prime}\right|_{[n]-B}=\left.T\right|_{[n]-B}$. It is clear from the definitions that $T^{\prime} \in L R T\left(\lambda ; R^{\prime}\right)$. The injection is given by $T \mapsto T^{\prime}$.

By composing embeddings of the form $\theta_{R}^{R^{\prime}}$ for $R \unrhd R^{\prime}$ a covering relation, one may obtain maps of the form $\theta_{R}^{R^{\prime}}$ for any pair $R \unrhd R^{\prime}$.

This conjecture is a consequence of the following.

Conjecture 18 Let $R \unrhd R^{\prime}$ be a covering relation. Then $\theta_{R}^{R^{\prime}}$ is independent of the sequence of covering relations in $\unrhd$ leading from $R$ to $R^{\prime}$, and

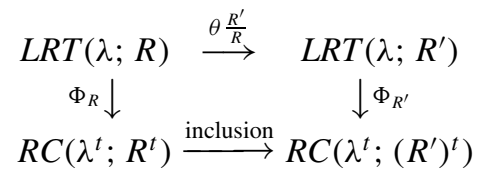

We conclude this section with a charge-preserving embedding

$$
\zeta: R C(\lambda ; R) \hookrightarrow R C(\lambda ; \operatorname{rows}(R))
$$

First we must define the charge of a rigged configuration and say a few words about quantum versus coquantum numbers. 
The charge of a rigged configuration $(v, J)$ is defined by

$$
\operatorname{charge}(v, J)=\operatorname{charge}(v)+\sum_{k \geq 1} \sum_{s=1}^{l\left(v^{k}\right)} J_{s}^{k}
$$

where

$$
\operatorname{charge}(v)=\sum_{k, n \geq 1}\left(\begin{array}{c}
\alpha_{k-1, n}-\alpha_{k, n}+\sum_{a}\left(\eta_{a}-k\right) \theta\left(\mu_{a}-n\right) \\
2
\end{array}\right)
$$

The definition of charge is compatible with the quantum number labelling in the following sense. Suppose $(\nu, L) \in R C(\lambda ; R)$; we view $L$ as coquantum numbers. Let $J$ be the rigging of $v$ obtained by complementing the rigging $L$, that is,

$$
J_{s}^{k}=P_{k, v_{s}^{k}}(v)-L_{s}^{k}
$$

Write $\Omega_{R}(\nu, L)=(\nu, J)$ for this involution. The $J$ are quantum numbers. The point is that

$$
\operatorname{charge}(v, J)+\operatorname{cocharge}(v, L)=n(R) .
$$

To see this, it is equivalent to show that

$$
\operatorname{charge}(v)=n(R)-\operatorname{cocharge}(v)-\sum_{k, n \geq 1} P_{k, n}(v) m_{n}\left(v^{k}\right)
$$

In light of (9.18) this is equivalent to

$$
\operatorname{charge}(v)=n(R)+\operatorname{cocharge}(v)+\sum_{k, n \geq 1} m_{k, n} r_{k, n} .
$$

where $\left(m_{k, n}\right)$ is the matrix associated to $v($ see $(9.2))$ and $r_{k, n}=r_{k, n}(R)$ is as in (2.8). Then

$$
\begin{aligned}
\operatorname{charge}(v) & =\sum_{k, n \geq 1}\left(\begin{array}{c}
m_{k, n}+r_{k, n} \\
2
\end{array}\right) \\
& =\sum_{k, n \geq 1}\left(\left(\begin{array}{c}
m_{k, n} \\
2
\end{array}\right)+\left(\begin{array}{c}
r_{k, n} \\
2
\end{array}\right)+m_{k, n} r_{k, n}\right) \\
& =\operatorname{cocharge}(v)+n(R)+\sum_{k, n} m_{k, n} r_{k, n}
\end{aligned}
$$

which proves (10.1). 
Now let us define the map $\zeta$. Let $v=\left(v^{1}, v^{2}, \ldots\right)$ be an admissible configuration of type $(\lambda ; R)$ and $J$ a rigging of $v$. Define $\zeta(v, J)=(\tilde{v}, \tilde{J})$ where

$$
\tilde{v}^{k}=v^{k} \bigcup_{a \geq 1}\left(\mu_{a}^{\left(\eta_{a}-k\right)_{+}}\right)
$$

and the quantum numbers on the "old" rows remain the same, and the "new" rows are assigned the quantum number 0 .

Proposition $13 \zeta: R C(\lambda ; R) \hookrightarrow R C(\lambda$; rows $(R))$ is a charge-preserving embedding.

This is an immediate consequence of the following description of $\zeta$.

Lemma 14 The map $\zeta$ satisfies the commutative diagram

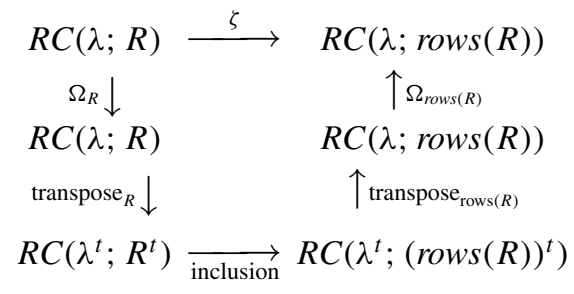

Proof: Let $(v, J) \in R C(\lambda ; R)$ and $(\tilde{v}, \tilde{J})$ the image of the composite map defined by the commutative diagram. Let $\Omega_{R}(v, J)=(v, L), \Omega_{\operatorname{rows}(R)}(\tilde{v}, \tilde{J})=(\tilde{v}, \tilde{L})$, and let the RC-transpose of $(v, L)$ be $(\hat{v}, \hat{L})$. Observe that the composite map is charge-preserving. We have

$$
\begin{aligned}
\operatorname{charge}(\tilde{v}, \tilde{J}) & =n(\operatorname{rows}(R))-\operatorname{cocharge}(\tilde{v}, \tilde{L}) \\
& =\operatorname{cocharge}(\hat{v}, \hat{L}) \\
& =n(R)-\operatorname{cocharge}(v, L) \\
& =\operatorname{charge}(\nu, J)
\end{aligned}
$$

by applications of (10.1) and (9.14) for both $R$ and $\operatorname{rows}(R)$.

So it suffices to show that the output $(\tilde{v}, \tilde{J})$ of the composite map agrees with $\zeta(v, J)$.

Let $\left(m_{i, j}\right),\left(\hat{m}_{i, j}\right)$, and $\left(\hat{t}_{i, j}\right)$ be the matrices (9.2) corresponding to $v, \hat{v}$, and $\tilde{v}$. Let $r_{i, j}=r_{i, j}(R)$ and $\tilde{r}_{i, j}=r_{i, j}(\operatorname{rows}(R))$ in the notation of (2.8). By two applications of the definition of the RC-transpose map (9.7) and the definition of $\operatorname{rows}(R)$, we have

$$
\begin{aligned}
\hat{m}_{i, j} & =-m_{j, i}+\theta\left(\lambda_{j}-i\right)-r_{j, i} \\
& =-\hat{t}_{j, i}+\theta\left(\lambda_{j}-i\right)-\tilde{r}_{j, i} .
\end{aligned}
$$


Solving for $\hat{t}_{j, i}$, we have

$$
\hat{t}_{j, i}=m_{j, i}+r_{j, i}-\tilde{r}_{j, i}
$$

By (9.3) we have

$$
\begin{aligned}
\tilde{\alpha}_{j, i} & =\alpha_{j, i}-\sum_{k=1}^{j} r_{j, i}-\tilde{r}_{j, i} \\
& =\alpha_{j, i}-\sum_{k=1}^{j} \sum_{a} \theta\left(\mu_{a}-i\right)\left(\theta\left(\eta_{a}-k\right)-\eta_{a} \delta_{1, k}\right) \\
& =\alpha_{j, i}-\sum_{a} \theta\left(\mu_{a}-i\right)\left(\min \left(\eta_{a}, j\right)-\eta_{a}\right) \\
& =\alpha_{j, i}+\sum_{a} \theta\left(\mu_{a}-i\right)\left(\eta_{a}-j\right)_{+} .
\end{aligned}
$$

This is equivalent to the assertion that the configuration in $\zeta(v, J)$ is equal to $\tilde{v}$.

To check the riggings, consider an index $k$ and a part size $n$. It is first shown that the following two assertions suffice.

1. If $m_{n}\left(v^{k}\right)>0$ and $P_{k, n}(v)>0$ then $\rho_{k, n}(v, J)=\rho_{k, n}(\tilde{v}, \tilde{J})$.

2. If $m_{n}\left(\tilde{v}^{k}\right)>0$ and $P_{k, n}(\tilde{v})>0$ then $\rho_{k, n}(\nu, J)=\rho_{k, n}(\tilde{v}, \tilde{J})$.

To see why these conditions suffice, let us consider a part $n$ of $\tilde{v}^{k}$, so that $m_{n}\left(\tilde{v}^{k}\right)>0$. Suppose first that this part $n$ is "new". If $P_{k, n}(\tilde{v})=0$ then $\tilde{J}$ obeys the definition of $\zeta$. So suppose $P_{k, n}(\tilde{v})>0$. Then by $2, \rho_{k, n}(v, J)=\rho_{k, n}(\tilde{v}, \tilde{J})$. Here the old parts of length $n$ in $\tilde{v}^{k}$ have the same labels as in $v^{k}$, using up all the nonzero parts of $\rho_{k, n}(\tilde{v}, \tilde{J})$. Therefore the new parts $n$ in $\tilde{v}^{k}$ have label zero, and $(\tilde{v}, \tilde{J})$ is as in the definition of $\zeta$. Otherwise suppose $n$ is an old part of $\tilde{v}^{k}$, so that $m_{n}\left(\tilde{v}^{k}\right)>0$ and $m_{n}\left(v^{k}\right)>0$. If either $P_{k, n}(v)>0$ or $P_{k, n}(\tilde{v})>0$ then by 1 or 2 we are done as before. If both are zero, then by admissibility the quantum number for this part must be zero in both $(\nu, J)$ and $(\tilde{v}, \tilde{J})$, and again we are done.

Now the proof of 2 is given; the proof of 1 is similar. For 2 , the hypotheses allow us to apply (9.11) for $(\tilde{v}, \tilde{L}) \in R C(\lambda ; \operatorname{rows}(R))$, so that $\rho_{n, k}(\hat{v}, \hat{L})$ is the complement of the transpose of $\rho_{k, n}(\tilde{v}, \tilde{L})$ in the $m_{n}\left(\tilde{v}^{k}\right) \times P_{k, n}(\tilde{v})$ rectangle. But by the definition of $\Omega_{\operatorname{rows}(R)}, \rho_{k, n}(\tilde{v}, \tilde{J})$ is the complement of $\rho_{k, n}(\tilde{v}, \tilde{L})$ in the $m_{n}\left(\tilde{v}^{k}\right) \times P_{k, n}(\tilde{v})$ rectangle. It follows that $\rho_{n, k}(\hat{v}, \hat{L})$ is the transpose of the partition $\rho_{k, n}(\tilde{v}, \tilde{J})$, and that $m_{k}\left(\hat{v}^{n}\right)>0$ and $P_{n, k}(\hat{v})>0$. Applying the same argument for $(\hat{v}, \hat{L}) \in R C\left(\lambda^{t} ; R^{t}\right)$ it follows that $\rho_{k, n}(v, L)$ is the transpose of the partition $\rho_{n, k}(\hat{v}, \hat{L})$, so that 2 follows.

It follows from the explicit description of $\zeta$ that we can embed the set $R C(\lambda ; R)$ into $R C(\lambda$; rows $(R))$ such that for each $k \geq 1$ and $a \geq 1$ there exists a nonnegative integer $m:=m(k, a)$ such that 
- $\alpha_{k, m+i}-\alpha_{k, m+i+1}=\mu_{a}$,

- $J_{m+i}^{k}=0$, for all $0 \leq i \leq\left(\eta_{a}-k\right)_{+}$.

If this characterization of the set $R C(\lambda ; R)$ is compared with that given by S. Fishel [7] for the set $M_{r-k}^{k}$, it follows immediately that

$$
M_{r-k}^{k}(t)=\sum_{(\nu, J) \in R C(\lambda ; R)} q^{\operatorname{charge}(v, J)}
$$

where $R=\left((2)^{r-k},(1,1)^{k},(1)^{n-2 r}\right)$. This, together with (2.4), implies that the Poincare polynomial $K_{\lambda ; R}(q)$ and the above generating function over rigged configurations, coincide in the case that $R$ consists of some rectangles (2) followed by a sequence of rectangles $(1,1)$ and (1).

\section{Appendix}

Before proving Lemma 11 it should be mentioned that the implication that 2 implies 1 is fundamental. It is used implicitly in the definition of the bijection from LR tableaux to rigged configurations, even in the Kostka case. So all the results depending upon that bijection require this lemma. Similarly, the definition of rigged configuration used in [7] requires this lemma.

Proof: (of Lemma 11) Clearly it is enough to show that 2 implies 1 and 1 implies 3. Suppose 2 holds. It is consistent with the definitions to make the convention that $P_{k, 0}(v)=0$ for all $k \geq 1$. Using the notation $\alpha_{k, n}$ for the $n$-th part of the transpose of the partition $v^{k}$, the difference of vacancy numbers is given by

$$
P_{k, n}(v)-P_{k, n-1}(v)=\alpha_{k-1, n}-2 \alpha_{k, n}+\alpha_{k+1, n}+\sum_{a} \theta\left(\mu_{a}-n\right) \delta_{\eta_{a}, k},
$$

which holds for $k, n \geq 1$. Taking differences again,

$$
\begin{aligned}
- & P_{k, n-1}(v)+2 P_{k, n}(v)-P_{k, n+1}(v) \\
& =\left(P_{k, n}(v)-P_{k, n-1}(v)\right)-\left(P_{k, n+1}(v)-P_{k, n}(v)\right) \\
& =m_{n}\left(v^{k-1}\right)-2 m_{n}\left(v^{k}\right)+m_{n}\left(v^{k+1}\right)+\sum_{a} \delta_{\mu_{a}, n} \delta_{\eta_{a}, k},
\end{aligned}
$$

valid for $k, n \geq 1$. In particular the vacancy numbers have the partial convexity property

$$
P_{k, n}(v) \geq 1 / 2\left(P_{k, n-1}(v)+P_{k, n+1}(v)\right) \quad \text { if } \quad m_{n}\left(v^{k}\right)=0 .
$$

So for 1 it is enough to show that $P_{k, n}(\nu) \geq 0$ for sufficiently large and small $n$. For small $n$, recall that $P_{k, 0}(v)=0$. For large $n, Q_{n}\left(v^{k}\right)=\left|v^{k}\right|$, so that the vacancy numbers 
satisfy

$$
\begin{aligned}
P_{k, n}(v)= & \left|v^{k-1}\right|-2\left|v^{k}\right|+\left|v^{k+1}\right|+\sum_{a} \min \left(\mu_{a}, n\right) \delta_{\eta_{a}, k} \\
= & \sum_{j>k-1} \lambda_{j}-\sum_{a \geq 1} \mu_{a}\left(\eta_{a}-(k-1)\right)_{+}-2\left(\sum_{j>k} \lambda_{j}-\sum_{a \geq 1} \mu_{a}\left(\eta_{a}-k\right)_{+}\right) \\
& +\sum_{j>k+1} \lambda_{j}-\sum_{a \geq 1} \mu_{a}\left(\eta_{a}-(k+1)\right)_{+}+\sum_{a} \min \left(\mu_{a}, n\right) \delta_{\eta_{a}, k} \\
= & \lambda_{k}-\lambda_{k+1}+\sum_{a \geq 1} \min \left(0, n-\mu_{a}\right) \delta_{\eta_{a}, k} .
\end{aligned}
$$

Thus for large $n, P_{k, n}(v) \geq \lambda_{k}-\lambda_{k+1} \geq 0$, proving 1 .

To prove 3 , note that

$$
\min \left(\lambda_{k}, n\right)-\min \left(\lambda_{k+1}, n\right)= \begin{cases}0 & \text { for } n \leq \lambda_{k+1} \\ n-\lambda_{k+1} & \text { for } \lambda_{k+1}<n<\lambda_{k} \\ \lambda_{k}-\lambda_{k+1} & \text { for } \lambda_{k} \leq n\end{cases}
$$

For $n \leq \lambda_{k+1}$ there is nothing to show. Suppose $n \geq \lambda_{k}$. In light of (11.2) it suffices to show that there is no index $a$ such that $\eta_{a}=k$ and $n<\mu_{a}$. Suppose such an $a$ exists. Then $\mu_{a}>n \geq \lambda_{k}$. In particular, the rectangle $R_{a}$ having $\eta_{a}=k$ rows and $\mu_{a}$ columns is not contained in the shape $\lambda$. By Theorem 4 it follows that there are no admissible configurations of type $(\lambda ; R)$, which is a contradiction.

Finally suppose $\lambda_{k+1}<n<\lambda_{k}$. In light of the partial convexity (11.1) and the boundary conditions $P_{k, \lambda_{k+1}}(v) \geq 0$ and $P_{k, \lambda_{k}}(v) \geq \lambda_{k}-\lambda_{k+1}$, it is enough to show that $m_{n}\left(v^{k}\right)=0$ for $n>\lambda_{k+1}$. Since $v^{k}$ is a partition it is enough to show $\lambda_{k+1} \geq v_{1}^{k}$ for all $k$. Fix $k$.

Observe first that for any $(\lambda ; R)$, if there is an admissible configuration of type $(\lambda ; R)$ then $\lambda_{1} \geq \mu_{a}$ for all $a$. Indeed, if such a configuration exists then by Theorem $4, L R_{\lambda}^{R}>0$, and by the LR rule there must be an $R$-LR tableau of shape $\lambda$. But for this to happen, $\lambda$ must contain $R_{i}$ for all $i$, hence $\lambda_{1} \geq \mu_{a}$ for all $a$.

Thus it suffices to exhibit a pair $(\tilde{\lambda} ; \tilde{R})$ such that $\tilde{\lambda}_{1}=\lambda_{k+1}$ and $\tilde{R}$ contains a rectangle having $v_{1}^{k}$ columns. Define the partition $\tilde{\lambda}$ and sequence of rectangles $\tilde{R}$ by

$$
\begin{aligned}
& \tilde{\lambda}=\left(\lambda_{k+1}, \lambda_{k+2}, \ldots\right) \\
& \tilde{R}=\left\{\left(\mu_{a}\right)^{\left(\eta_{a}-k\right)_{+}}\right\} \cup\left\{\left(v_{b}^{k}\right) \mid 1 \leq b \leq \ell\left(v^{k}\right)\right\} .
\end{aligned}
$$

In other words, $\tilde{\lambda}$ is obtained from $\lambda$ by removing the first $k$ parts, and $\tilde{R}$ is obtained from $R$ by removing $k$ rows from each rectangle and adding a single row for each part of the partition $v^{k}$. Now it is enough to exhibit an admissible configuration of type $(\tilde{\lambda} ; \tilde{R})$. Recall that we are assuming that $v$ is an admissible configuration of type $(\lambda ; R)$. Let $\tilde{v}$ be the sequence of partitions defined by $\tilde{v}^{p}=v^{k+p}$ for $p \geq 1$. Let us check the condition that $\tilde{v}$ 
is a configuration of type $(\tilde{\lambda} ; \tilde{R})$. For $p \geq 1$,

$$
\begin{aligned}
\left|\tilde{v}^{p}\right| & =\left|v^{k+p}\right| \\
& =\sum_{j>k+p} \lambda_{j}-\sum_{a} \mu_{a} \max \left(\eta_{a}-k-p, 0\right) \\
& =\sum_{j>p} \tilde{\lambda}_{j}-\sum_{a} \mu_{a} \max \left(\left(\eta_{a}-k\right)_{+}-p, 0\right)-\sum_{1 \leq b \leq \ell\left(v^{k}\right)} v_{b}^{k} \max (1-p, 0) .
\end{aligned}
$$

To check that $\tilde{v}$ is admissible, let $n \geq 1$ and $p>1$ first:

$$
\begin{aligned}
P_{p, n}(\tilde{v})= & Q_{n}\left(\tilde{v}^{p-1}\right)-2 Q_{n}\left(\tilde{v}^{p}\right)+Q_{n}\left(\tilde{v}^{p+1}\right)+\sum_{a} \min \left(\mu_{a}, n\right) \delta_{\left(\eta_{a}-k\right)_{+}, p} \\
& +\sum_{b} \min \left(v_{b}^{k}, n\right) \delta_{1, p} \\
= & Q_{n}\left(v^{k+p-1}\right)-2 Q_{n}\left(v^{k+p}\right)+Q_{n}\left(v^{k+p+1}\right)+\sum_{a} \min \left(\mu_{a}, n\right) \delta_{\eta_{a}, k+p} \\
= & P_{k+p, n}(v) \geq 0
\end{aligned}
$$

by the admissibility of $\nu$. For $p=1$ and $n \geq 1$ we have

$$
\begin{aligned}
P_{1, n}(\tilde{v})= & Q_{n}\left(\tilde{v}^{0}\right)-2 Q_{n}\left(\tilde{v}^{1}\right)+Q_{n}\left(\tilde{v}^{2}\right)+\sum_{a} \min \left(\mu_{a}, n\right) \delta_{\left(\eta_{a}-k\right)_{+}, 1} \\
& +\sum_{b} \min \left(v_{b}^{k}, n\right) \delta_{1,1} \\
= & 0-2 Q_{n}\left(v^{k+1}\right)+Q_{n}\left(v^{k+2}\right)+\sum_{a} \min \left(\mu_{a}, n\right) \delta_{\eta_{a}, k+1}+Q_{n}\left(v^{k}\right) \\
= & P_{k+1, n}(v) \geq 0
\end{aligned}
$$

again by the admissibility of $\nu$. Thus $\tilde{v}$ is an admissible configuration of type $(\tilde{\lambda} ; \tilde{R})$ and the proof is complete.

\section{References}

1. A. Broer, "Normality of some nilpotent varieties and cohomology of line bundles on the cotangent bundle of the flag variety," in Lie Theory and Gometry, Progr. Math., Vol. 123, Birkhäuser Boston, Boston, MA, 1994, pp. 1-19.

2. A. Broer, personal communication, 1995.

3. A.D. Berenstein and A.N. Kirillov, "Groups generated by involutions, Gelfand-Tsetlin patterns and combinatorics of Young tableaux," St. Petersburg Math. J. 7 (1996), 77-127.

4. L.M. Butler, "Combinatorial properties of partially ordered sets associated with partitions and finite Abelian groups," Ph.D. Thesis, MIT, May 1986.

5. I.F. Donin, "Multiplicities of $S_{n}$ modules and the Index and Charge of tableaux," Func. Anal. App. 27 (1994), 280-282.

6. W. Fulton, "Young tableaux," London Mathematical Society Student Texts, Vol. 35, Cambridge University Press, Cambridge, 1997.

7. S. Fishel, "Statistics for special q, t-Kostka polynomials," Proc. Amer. Math. Soc. 123 (1995), 2961-2969. 
8. S.V. Fomin and C. Greene, "A Littlewood-Richardson miscellany,” Eur. J. Combin. 14 (1993), 191-212.

9. R.K. Gupta, "Generalized exponents via Hall-Littlewood symmetric functions," Bull. AMS 16 (1987), 287291.

10. A.N. Kirillov, "Bethe ansatz and combinatorics of Young tableaux" Ph.D. Thesis, LOMI, Leningrad, 1987, (in Russian).

11. A.N. Kirillov, "On the Kostka-Green-Foulkes polynomials and Clebsch-Gordan numbers," J. Geom. Phys. 5 (1988), 365-389.

12. A.N. Kirillov, "Ubiquity of Kostka polynomials," in Proceedings of the Nagoya 1999 International Workshop on Physics and Combinatorics (Physics and Combinatorics 1999) Nagoya University, August 23-27, 1999, pp. 85-200, World Scientific, Singapore, 2001.

13. A.N. Kirillov, "Combinatorics of Young tableaux and configurations," Proceedings of the St. Petersburg Mathematical Society, Vol. VII, pp. 17-98, Amer. Math. Soc. Transl. Ser. 2, Vol. 203, Amer. Math. Soc., Providence, RI, 2001.

14. J. Klimek, W. Kraśkiewicz, M. Shimozono, and J. Weyman, "On the Grothendieck group of modules supported in a nilpotent orbit in the Lie algebra gl(n)," J. Pure Appl. Algebra 153 (2000), 237-261.

15. A.N. Kirillov, A. Lascoux, B. Leclerc, and J.-Y. Thibon, "Séries génératrices pour les tableaux de dominos," C. R. Acad. Sci. Paris Sér. I Math. 318 (1994), 395-400.

16. A.N. Kirillov, A. Schilling, and M. Shimozono, "A bijection between Littlewood-Richardson tableaux and rigged configurations," Selecta Math. N. S., to appear (math.CO/9901037).

17. D.E. Knuth, "Permutations, matrices, and generalized Young tableaux," Pacific J. Math. 34 (1970), 709-727.

18. A.N. Kirillov and N.Y. Reshetikhin, "The Bethe Ansatz and the combinatorics of Young tableaux," J. Soviet Math. 41 (1988), 925-955.

19. A. Lascoux, "Cyclic permutations on words, tableaux and harmonic polynomials," Proc. of the Hyderabad Conference on Algebraic Groups, 1989, pp. 323-347, Manoj Prakashan, Madras, 1991.

20. A. Lascoux, B. Leclerc, and J.-Y. Thibon, "Ribbon tableaux, Hall-Littlewood functions, quantum affine algebras, and unipotent varieties," J. Math. Phys. 38 (1997), 1041-1068.

21. A. Lascoux, B. Leclerc, and J.-Y. Thibon, "Crystal graphs and $q$-analogues of weight multiplicities for root systems of type $A_{n}$, , Lett. Math. Phys. 39 (1995), 359-374.

22. D.E. Littlewood and A.R. Richardson, "Group characters and algebra," Phil. Trans. Royal Soc. London Ser A 233 (1934), 99-141.

23. A. Lascoux and M.-P. Schützenberger, "Sur une conjecture de H. O. Foulkes," C. R. Acad. Sc. Paris, 286A (1978), 323-324.

24. A. Lascoux and M.P. Schützenberger, "Croissance des polynômes de Foulkes/Green,” C. R. Acad. Sc. Paris 288A (1979), 95-98.

25. A. Lascoux and M.P. Schützenberger, "Le monoïde plaxique," in Noncommutative Structures in Algebra and Geometric Combinatorics, A. de Luca (Ed.), Quaderni della Ricerca Scientifica del C. N. R., Roma, 1981, pp. $129-156$.

26. B. Leclerc and J.-Y. Thibon, "Littlewood-Richardson coefficients and Kazhdan-Lusztig polynomials," Adv. Studies in Pure Math. 28 (2000), 155-220.

27. I.G. MacDonald, Symmetric Functions and Hall Polynomials, Oxford Univ. Press, Oxford, 1979.

28. I.G. MacDonald, Notes on Schubert Polynomials, LACIM, Montréal, 1991.

29. A.O. Morris, "The characters of the group GL(n, q)," Math. Zeitschr. 81 (1963), 112-123.

30. M. Okado, A. Schilling, and M. Shimozono, "Virtual crystals and fermionic formulas of type $D_{n+1}^{(2)}, A_{2 n}^{(2)}$, and $C_{n}^{(1)}$, , preprint math.QA/0105017, submitted.

31. V. Reiner and M. Shimozono, "Specht series for column convex diagrams," J. Algebra, 174 (1995), 489-522.

32. J.B. Remmel and R. Whitney, "Multiplying Schur functions," J. Algorithms 5 (1984), 471-487.

33. B. Sagan, The Symmetric Group: Representations, Combinatorial Algorithms, and Symmetric Functions, Wadsworth \& Brooks/Cole, Pacific Grove, 1991.

34. C. Schensted, "Longest increasing and decreasing sequences," Canad. J. Math. 13 (1961), 179-191.

35. A. Schilling and S.O. Warnaar, "Inhomogenous lattice paths, generalized Kostka polynomials and $A_{n-1}$ supernomials," Commun. Math. Phys. 202 (1999), 359-401.

36. M. Shimozono, "Littlewood-Richardson rules for ordinary and projective representations of symmetric groups," Ph.D. Thesis, University of California, San Diego, September 1991. 
37. M. Shimozono, “A cyclage poset structure for Littlewood-Richardson tableaux,” Europ. J. Combin. 22 (2001), 365-393.

38. M. Shimozono, "Multi-atoms and monotonicity of generalized Kostka polynomials," Europ. J. Combin. 22 (2001), 395-414.

39. M. Shimozono, "Affine type A crystal structure on tensor products of rectangles, Demazure characters, and nilpotent varieties," preprint math.QA/9804039.

40. J. Stembridge, "Some particular entries of the two-parameter Kostka matrix," Proc. Amer. Math. Soc. 121 (1994), 367-373.

41. M. Shimozono and J. Weyman, "Graded characters of modules supported in the closure of a nilpotent conjugacy class," European J. Combin. 21 (2000), 257-288.

42. D. Stanton and D. White, "A Schensted algorithm for rim hook tableaux," J. Combin. Th. Ser. A 40 (1985), 211-247.

43. J. Weyman, "The equations of conjugacy classes of nilpotent matrices," Invent. Math. 98 (1989), 229-245.

44. D.E. White, "Some connections between the Littlewood-Richardson rule and the construction of Schensted," J. Comb. Th. Ser. A 30 (1981), 237-247. 